\title{
Uplink interference protection and scheduling for energy efficient OFDMA networks
}

\author{
Harald Burchardt ${ }^{1 *}$, Zubin Bharucha ${ }^{2}$, Gunther Auer ${ }^{2}$ and Harald Haas ${ }^{1}$
}

\begin{abstract}
One of the key challenges for future orthogonal frequency division multiple access-based networks is inter-cell interference coordination. With full frequency reuse and small inter-site distances, coping with co-channel interference $(\mathrm{CCl})$ in such networks has become increasingly important. In this article, an uplink interference protection (ULIP) technique to combat CCI is introduced and investigated. The level of uplink interference originating from neighbouring cells (affecting co-channel mobile stations (MSs) in the cell of interest) can be effectively controlled by reducing the transmit power of the interfering MSs. This is done based on the target signal-to-noise-plus-interference ratio (SINR) and tolerable interference of the vulnerable link. Bands are prioritised in order to differentiate those (vulnerable/victim) MSs that are to be protected from interference and those (aggressor/interfering MSs) that are required to sacrifice transmission power to facilitate the protection. Furthermore, MSs are scheduled such that those users with poorer transmission conditions receive the highest interference protection, thus balancing the areal SINR distribution and creating a fairer allocation of the available resources. In addition to interference protection, the individual power reductions also serve to decrease the total system uplink power, resulting in a greener system. It is shown through analytic derivation that the introduction of ULIP guarantees an increase in energy efficiency for all MSs, with the added benefit that gains in overall system throughput are also achievable. Extensive system level simulations validate these findings.
\end{abstract}

Keywords: inter-cell interference coordination, uplink interference protection, OFDMA networks, fair scheduling

\section{Introduction}

In wireless networks, there is an increasing demand for higher user and system throughput, along with growing expectation for all mobile stations (MSs) in a cell to be capable of supporting data-heavy multimedia and Internet services. This is especially difficult to maintain at the celledge, where received signal and service clearly deteriorate. Furthermore, the necessity for more energy efficient, or "green," technologies is growing. With base stations (BSs) requiring up to $1.5 \mathrm{~kW}$, a typical wide area network can consume tens of MW per annum [1]. In the uplink, while MSs do not consume nearly as much power, there are orders of magnitude more MSs then BSs in the network [2]. In addition with traffic loads increasing approximately ten times every 5 years, a doubling of the energy consumption results over the same time period. Clearly, such

\footnotetext{
* Correspondence: h.burchardt@ed.ac.uk

${ }^{1}$ Institute for Digital Communications, School of Engineering and Electronics, The University of Edinburgh, EH9 3JL, Edinburgh, UK

Full list of author information is available at the end of the article
}

an increase raises serious environmental concerns. Consequently, smaller cell sizes, femto-cell deployment, relays $[3,4]$ and especially inter-cell interference coordination (ICIC) techniques are envisioned for future wireless networks to improve user throughputs and network energy efficiency, while sacrificing minimal system capacity.

For future wireless networks, such a reduction in cell size is undertaken due to transmit power limitations and constraints on the link budget [5]. The demand for higher data rates coupled with full frequency reuse results in an interference-limited system, which cannot achieve full capacity without the implementation of one or more viable interference mitigation/cancellation/coordination techniques [5]. Furthermore, through the implementation of orthogonal frequency division multiple access (OFDMA) in the downlink and single carrier frequency division multiple access (SC-FDMA) in the uplink as multiple access schemes, future systems will provide orthogonality between resource blocks (RBs) in both directions, and hence also between all users within 
a cell [2]. Thus, system performance is mainly limited by interference originating from users in neighbouring cells, which can be detrimental to the signal-to-noise-plusinterference ratio (SINR) and throughput performance of MSs using the same RBs [6]. A typical solution is to force interferers to leave those RBs idle. However, this severely harms the trunking efficiency of the network [7]. Hence, suppressing transmission is clearly suboptimal, and thus interference coordination techniques are necessary to achieve desired sum and individual throughputs.

For OFDMA systems, some traditional ICIC techniques, such as power control, interference cancellation, fractional frequency reuse, multiple-input multipleoutput transmission and space division multiple access [2], have been proposed. Some of these strategies, however, require knowledge about the position of a MS relative to it's own and neighbouring BSs [2], which clearly increases the signalling burden in the network. In [8], other specific ICIC techniques are suggested, such as slow power control, frequency division multiplexing resource allocation, and coordination by MS alignment, though management of interference from other cells is not considered. Further research in [9] presents a distributed uplink power allocation technique based on a maximum sum rate optimisation, yielding superior results in terms of average system throughput, however ignoring the tradeoff between cell-edge performance and overall spectral efficiency. In [10], a softer frequency reuse scheme is introduced, where cell-edge power masks are used to mitigate inter-cell interference. These fixed masks cannot, however, adapt to the service-dependent requirements of the neighbouring cells, potentially wasting bandwidth. In [11], the downlink scheduling is formulated as an optimisation problem, and a decomposition of the problem is performed. Here, however, cochannel interference (CCI) (in future systems from neighbouring cells) is not taken into account, and hence the scheduling becomes suboptimal for multiple access channels and large networks.

In [12], a dynamic channel acquisition algorithm based on convex optimisation for the wireless downlink is considered, which provides optimal power and throughput performance for i.i.d. channels. This optimality suffers however for general ergodic channels, and hence is not suitable for mobile environments. In [13], the authors propose a low-complexity algorithm with fairness consideration to optimise the sum rate under individual rate and power constraints. Here though, because the water-filling solution is used for rate-optimal power allocation, a fair power distribution is neglected. In [14], an optimisationbased heuristic inter-cell coordination scheme is proposed to regulate the uplink transmission in neighbouring cells such that inter-cell interference is mitigated. As the scheme operates iteratively on a two-cell basis, however, it is clearly unsuitable for multi-cellular resource allocation. Finally, in [15], an energy-aware cross-layer radio management framework is proposed, that partitions the global optimisation problem into subproblems, which can be solved locally. While achieving substantial gains, the focus of the work is on multimode communication (i.e., cellular, WLANs, WMANs, etc.), and so an optimisation for pure cellular communication is not offered. In general, it is evident that the challenge of resource and power allocation has been thoroughly investigated as an optimisation problem, however in most cases these problems are non-convex, very hard to solve, and hence suboptimal heuristics are developed. In this work, a resource and power allocation technique based on local interference requirements will be developed to manage this challenge.

Much of the previous work on energy efficient systems concentrates on network optimisation and scheduling policies. Macro-cell size reduction for better energy efficiency is investigated in [16], with positive results. Of course, reducing the cell-sizes means increasing the number of BSs in an area, which is generally rejected due to the enhanced infrastructure expenses. In [17], game-theoretic approaches are utilised to, minimise the cost per reliable bit sent in energy constrained networks. However, it is seen that there is a clear tradeoff between energy and spectral efficiency, and hence the energy-efficient resource allocations tend to be spectrally inefficient. This is further highlighted in [18], where an analytical model determines the optimal energy-spectral efficiency tradeoff for the downlink in OFDMA networks. In this article, however, we present an ICIC technique which utilises interfering link gains to not only provide interference mitigation and spectral efficiency gains in the uplink, but also generate large energy savings.

An energy efficient interference protection technique for the uplink of OFDMA-based systems is introduced. By reducing the power on the interfering link, the SINRs of individual RBs can be enhanced. This power reduction also results in a more energy efficient system. By segregating the spectrum into priority bands, MSs allocated lower priority RBs provide interference protection for higher priority RBs in neighbouring cells by decreasing their transmit power. The priority bands (i.e., low to high) are allocated such that the same RBs in any neighbouring cells do not share the same priority class, and hence a priority reuse scheme [19] is established. Furthermore, the proposed power reduction is based on target SINRs, providing real-time service-dependent interference coordination and energy efficiency in the uplink.

The rest of the article is structured as follows: Section 2 describes the system and channel environment, Section 3 explains the uplink interference protection (ULIP) proto$\mathrm{col}$ and its performance in wireless networks is analysed 
in Section 4. In Sections 5 and 6 the resource scheduler and simulation are described, respectively. Finally, Section 7 portrays and discusses the simulation results, and some concluding remarks are offered in Section 8.

\section{System and channel model $B / M$}

The reverse link of an OFDMA system is considered, where the system bandwidth $B$ is divided into $M$ RBs. A $R B$ defines one basic time-frequency unit of bandwidth $B_{\mathrm{RB}}=B / \mathrm{M}$. All MSs can transmit up to a maximum power $P_{\max }$, and hence up to $P_{\max } / M$ on each RB. Perfect time and frequency synchronisation is assumed.

Universal frequency reuse is considered, so that each macro-cell utilises the entire system bandwidth $B$. The set of RBs $\mathcal{M}$, where $|\mathcal{M}|=M$, is distributed by each BS to its associated MSs. Throughout this article, $u$ is used to define any MS, and $v_{u}$ the BS with which this MS is associated. The received signal observed by $\mathrm{BS}_{v_{u}}$ from $\mathrm{MS}_{u}$ on $\mathrm{RB}_{m}$ is given by

$$
Y_{u}^{m}=\underbrace{P_{u}^{m} G_{u, u_{u}}^{m}}_{S_{u}^{m}}+I_{u}^{m}+\eta,
$$

where $G_{u, v_{u}}^{m}$ denotes the channel gain between the $\mathrm{MS}_{u}$ and its serving $\mathrm{BS}_{v_{u}}$, observed on $\mathrm{RB}_{m}$. Furthermore, $P_{u}^{m}$ denotes the transmit power of $\mathrm{MS}_{u}$ on $\mathrm{RB}_{m}$, $S_{u}^{m}$ the desired received signal, $\eta$ the thermal noise, and $I_{u}^{m}$ the CCI received on $\mathrm{RB}_{m}$ from MSs in neighbouring cells. The interference $I_{u}^{m}$ is defined by

$$
I_{u}^{m}=\sum_{i \in \mathcal{I}_{m}} P_{i}^{m} G_{i, v_{u^{\prime}}}^{m}
$$

where $\mathcal{I}_{m}$ represents the set of interferers (i.e., the set of MSs in neighbouring cells that are also assigned $\left.\mathrm{RB}_{m}\right)$. Hence, the SINR observed at the $\mathrm{BS}_{v_{u}}$ on $\mathrm{RB}_{m}$ is calculated by

$$
\gamma_{u}^{m}=\frac{S_{u}^{m}}{I_{u}^{m}+\eta}=\frac{P_{u}^{m} G_{u, v_{u}}^{m}}{\sum_{i \in \mathcal{I}_{m}} P_{i}^{m} G_{i, v_{u}}^{m}+\eta} .
$$

The achievable throughput on the link between $\mathrm{MS}_{u}$ and $\mathrm{BS}_{v_{u}}$ on $\mathrm{RB}_{m}$ using adaptive modulation and coding (AMC) is given by

$$
C_{u}^{m}\left(\gamma_{u}^{m}\right)=k_{\mathrm{sc}} \varrho_{\mathrm{s}} \varepsilon_{\mathrm{s}}\left(\gamma_{u}^{m}\right) \quad\left[\frac{\mathrm{bits} / \mathrm{s}}{\mathrm{RB}}\right],
$$

where $k_{\mathrm{sc}}$ is the number of subcarriers per RB, $\varrho_{\mathrm{s}}$ the symbol rate per subcarrier, and $\varepsilon_{s}\left(\gamma_{u}^{m}\right)$ the symbol efficiency given in Table $1 .^{\mathrm{a}}$
Table 1 Adaptive modulation and coding table

\begin{tabular}{lllll}
\hline $\begin{array}{l}\text { CQI } \\
\text { index }\end{array}$ & $\begin{array}{l}\text { min. SINR } \\
{[\mathrm{dB}]}\end{array}$ & Modulation & $\begin{array}{l}\text { Code } \\
\text { rate }\end{array}$ & $\begin{array}{l}\text { Efficiency } \boldsymbol{\varepsilon}_{\mathbf{s}} \text { [bits/ } \\
\text { sym] }\end{array}$ \\
\hline 0 & - & None & - & 0 \\
1 & -6 & QPSK & 0.076 & 0.1523 \\
2 & -5 & QPSK & 0.12 & 0.2344 \\
3 & -3 & QPSK & 0.19 & 0.3770 \\
4 & -1 & QPSK & 0.3 & 0.6016 \\
5 & 1 & QPSK & 0.44 & 0.8770 \\
6 & 3 & QPSK & 0.59 & 1.1758 \\
7 & 5 & 16QAM & 0.37 & 1.4766 \\
8 & 8 & 16QAM & 0.48 & 1.9141 \\
9 & 9 & 16QAM & 0.6 & 2.4063 \\
10 & 11 & 64QAM & 0.45 & 2.7305 \\
11 & 12 & 64QAM & 0.55 & 3.3223 \\
12 & 14 & 64QAM & 0.65 & 3.9023 \\
13 & 16 & 64QAM & 0.75 & 4.5234 \\
14 & 18 & 64QAM & 0.85 & 5.1152 \\
15 & 20 & 64QAM & 0.93 & 5.5547 \\
\hline
\end{tabular}

Further, $C_{u}$ denotes the achievable throughput of $\mathrm{MS}_{u}$, and is calculated by the aggregate throughput achieved on the RBs assigned to $\mathrm{MS}_{u}$

$$
C_{u}=\sum_{m \in \mathcal{M}_{u}} C_{u}^{m}=\sum_{m \in \mathcal{M}_{u}} k_{\mathrm{sc}} \varrho_{\mathrm{s}} \varepsilon_{\mathrm{s}}^{m} \quad\left[\frac{\text { bits }}{\mathrm{s}}\right],
$$

where $\mathcal{M}_{u}$ describes the set of RBs assigned to $\mathrm{MS}_{u}$ in the current transmission, and $\varepsilon_{\mathrm{s}}^{m}=\varepsilon_{\mathrm{s}}\left(\gamma_{u}^{m}\right)$. Finally, the system capacity is calculated as the sum of achievable throughput of all MSs

$$
C_{\text {sys }}=\sum_{u} C_{u} .
$$

The energy efficiency $\beta_{u}$ measures the data sent per unit of energy (or, alternatively, data rate per unit of transmit power) of $\mathrm{MS}_{u}$. This is defined as follows:

$$
\beta_{u}=\frac{C_{u}}{P_{u}}=\frac{\sum_{m \in \mathcal{M}_{u}} k_{\mathrm{sc}} \varrho_{s} \varepsilon_{\mathrm{s}}^{m}}{\sum_{m \in \mathcal{M}_{u}} P_{u}^{m}}\left[\frac{\text { bits } / \mathrm{s}}{\mathrm{W}}\right] \equiv\left[\frac{\text { bits }}{\mathrm{J}}\right],
$$

where $P_{u}$ is the total transmit power of $\mathrm{MS}_{u}$, and $C_{u}$ the throughput from (5).

Lastly, Jain's fairness index [20] is used to calculate the throughput fairness of the system in each time slot (i.e., Long-Term Evolution (LTE) subframe)

$$
\Gamma(k)=\frac{\left[\sum_{u} C_{u}(k)\right]^{2}}{N_{\text {sys }} \sum_{u} C_{u}(k)^{2}} .
$$

where $k$ indicates the time slot, $N_{\text {sys }}$ the number of MS in the system, and $C_{u}(k)$ the achieved throughput of $\mathrm{MS}_{u}$ over all time slots 1: $k$. 


\subsection{Channel model}

In general, the channel gain, $G_{k, l}^{m}$, between a transmitter $k$ and receiver $l$, observed on $\mathrm{RB}_{m}$ and separated by $d \mathrm{~m}$ is determined by the path loss, log-normal shadowing, and channel variations caused by frequency-selective fading:

$$
G_{k, l}^{m}=\left|H_{k, l}^{m}\right|^{2} 10^{\frac{-L(d)+X \sigma}{10}},
$$

where $H_{k, l}^{m}$ describes the channel transfer function between transmitter $k$ and receiver $l$ on $\mathrm{RB}_{m}, L(d)$ is the distance-dependent path loss (in $\mathrm{dB}$ ) and $X_{\sigma}$ is the lognormal shadowing value (in $\mathrm{dB}$ ) with standard deviation $\sigma$, as described in [21]. The channel generally exhibits time and frequency dispersions, however channel fluctuations within a $\mathrm{RB}$ are not considered as the RB dimensions are significantly smaller than the coherence time and frequency of the channel [22]. Furthermore, the large-scale path loss $L(d)$ is identical on all RBs assigned to a MS. Finally, the delay profiles used to generate the frequencyselective fading channel transfer factor $H_{k, l}^{m}$ are taken from applicable propagation scenarios in $[21,23]$.

The path loss model used to calculate $L(d)$ is for a purely outdoor link [24], i.e., the link (desired or interfering) between a BS and an outdoor MS, and calculates the path loss as

$$
L(d)=15.3+37.6 \log _{10}(d) \quad[\mathrm{dB}]
$$

where $d$ is the distance between transmitter and receiver.

Log-normal shadowing is added to all links through the use of correlated shadowing maps. These are generated such that the correlation between two points is distancedependent.

\section{Uplink interference protection (ULIP)}

Traditional uplink power control methods use the estimated path gain on the intended link to perform MS transmit power adaptation [25,26]. A better option is to utilise the interfering link, i.e., to a neighbouring BS, to reduce the transmit power on the affected RBs, such that interference caused to neighbouring BSs is lessened. This way, vulnerable MSs in the cell of interest have a chance of maintaining sufficient SINR, while the offending links remain active.

\subsection{Uplink interference scenario}

Figure 1 portrays the interference scenario of two MSs in the uplink.

Here, the vulnerable $\mathrm{MS}_{v}$ served by $\mathrm{BS}_{v_{v}}$ and the interfering $\mathrm{MS}_{i}$ served by $\mathrm{BS}_{v_{i}}$ are transmitting on the same $\mathrm{RB}$. Due to the uplink interference at $\mathrm{BS}_{v_{v}}$ caused by $\mathrm{MS}_{i}$, the SINR of $\mathrm{MS}_{v}$ may fall below the SINR target, $\gamma_{v}^{\mathrm{tar}}$. To

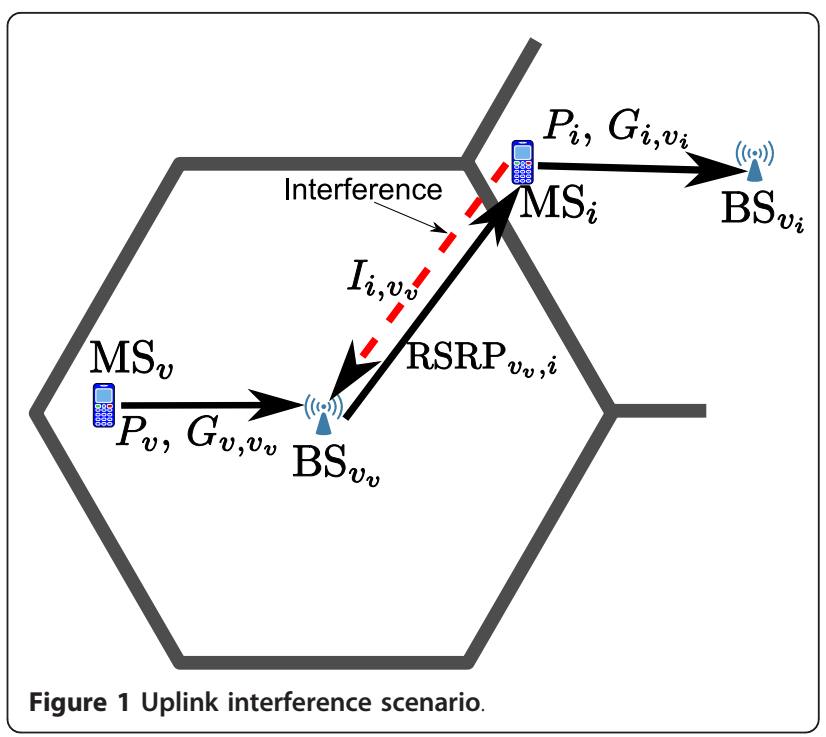

prevent such a situation, an interference protection technique is devised that reduces the transmit power $P_{i}$ such that $\mathrm{MS}_{v}$ achieves a satisfactory SINR, $\gamma_{v} \geq \gamma_{v}^{\mathrm{tar}}$.

\subsection{Interference aware power reduction}

The goal is to find an effective method to scale the transmit power on the interfering RBs. Here, the downlink reference signal of the neighbouring cells aid the MS in estimating the interference it causes to the neighbouring cells, assuming channel reciprocity. The channel can be considered reciprocal in terms of path loss and shadowing, however fast fading reciprocity is not assumed as this is not always the case, especially in frequency division duplex (FDD) systems. For LTE, the reference signal received power (RSRP) in particular is used. The RSRP provides a cell-specific signal strength metric. It is used mainly to rank different cells according to signal strength and to perform handover and cell reselection decisions [27]. The reference signals facilitate the adaptation of the interfering RB transmit power, which is performed as follows:

(1) Assume $\mathrm{MS}_{v}$ has been allocated the vulnerable $\mathrm{RB}_{m}$. Let $\gamma_{v}^{\mathrm{tar}}$ be the known, service-dependent target SINR of $\mathrm{MS}_{v}$, calculated as

$$
\gamma_{v}^{\mathrm{tar}}=\frac{P_{v}^{m} G_{v, v_{v}}^{m}}{I_{v}^{m, \text { tol }}+N}
$$

where $P_{v}^{m}$ is the transmit power on $\mathrm{RB}_{m}, G_{v, v_{v}}^{m}$ is the path gain between $\mathrm{MS}_{v}$ and its $\mathrm{BS}_{v_{v}}$, and $I_{v}^{m \text {,tol }}$ is the tolerable interference such that $\gamma_{v}^{\text {tar }}$ can be met on $\mathrm{RB}_{m}$.

(2) Considering RSRPs of the neighbouring cells; as any reference signal is transmitted at a fixed power, an interfering $\mathrm{MS}_{i}$ can calculate the path gain on $\mathrm{RB}_{m}$, 
, to the affected $\mathrm{BS}_{v_{v}}$, and assuming channel reciprocity, estimate the interference it is causing. It then uses the $I_{v}^{m, \text { tol }}$ from the vulnerable MS to calculate the maximum power, $\tilde{P}_{\max , i}$, for $\mathrm{MS}_{i}$ as

$$
\tilde{P}_{\max , i}=\frac{I_{v}^{m, t o l}}{G_{v_{v}, i}^{m}} .
$$

It is clear that $\tilde{P}_{\max , i}$ is directly proportional to the tolerable interference, $I_{v}^{m, \text { tol }}$ at $\mathrm{MS}_{v}$.

Given the power adaptation scheme and assuming channel reciprocity, $\mathrm{MS}_{v}$ should achieve the required SINR target on $\mathrm{RB}_{m}$. However, in a FDD system where fast fading is not reciprocal, an interference margin must be applied. Lastly, since $I_{v}^{m, \text { tol }}$ is not directly available at $\mathrm{MS}_{i}$, this needs to be signalled from $\mathrm{BS}_{v_{v}}$ to $\mathrm{MS}_{i}$ via existing backhaul infrastructures.

\subsection{Priority bands}

In [19], soft frequency reuse, where RBs are arranged into priority bands, is envisioned for LTE systems to facilitate interference protection. In this work, the available spectrum is split into different priority classes. ${ }^{\mathrm{b}} \mathrm{RBs}$ assigned high-priority status are allocated to those MSs that require interference protection, and hence do not need to scale their transmit power. Looking from the other perspective, strongly interfering MSs are allocated RBs with a low-priority status, such that the transmit powers on these RBs may be reduced to provide interference protection. A priority class reuse scheme is established which, due to the power reduction, is an adaptive form of softer frequency reuse [10].

Three bands of communication, termed high-priority, mid-priority, and low-priority, are defined. These bands are allocated orthogonally, such that if a RB is assigned high-priority status in one cell, the same RB is assigned mid-priority and low-priority status in the neighbouring cells. In this sense, a priority class reuse factor of three results, which is shown in Figures 2 and 3.

When excessive interference is caused, the owners of mid- and low-priority RBs in the neighbouring cells must reduce their transmit power. This boosts the SINR on both the high-and mid-priority RBs. The power reduction procedure for ULIP is performed as follows:

(1) The $I_{v}^{m, \text { tol }}$ for the high-priority RBs are calculated from (11), and distributed to the neighbouring cells.

(2) The transmit powers on mid-priority RBs are adjusted according to (12) based on the $\min _{v \in \mathcal{I}_{m}}\left\{I_{v}^{m, \text { tol }}\right\}$ received from high-priority RBs in neighbouring cells.

(3) The $I_{v}^{m, \text { tol }}$ for the mid-priority RBs (after power scaling has been performed) are calculated from (11) and distributed.

(4) The transmit powers on low-priority RBs are adjusted based on $\min _{v \in \mathcal{I}_{m}}\left\{I_{v}^{m, \text { tol }}\right\}$ received from both neighbouring high- and mid-priority RBs.

It is clear that $I_{v}^{m, \text { tol }}$ can be re-calculated in every time slot. However, to reduce the signaling burden on the network, these updates are only distributed when a sufficient difference, $\delta$, to the last sent $I_{v}^{m, \text { tol }}$ has been observed.

Furthermore, all high-priority RBs receive interference protection, and consequently gains in achievable throughput. This is facilitated by the MSs assigned lowand mid-priority RBs, which have reduced their transmit power. MSs allocated mid-priority RBs may also receive a throughput boost, as the MSs assigned low-priority RBs also take the mid-priority $I_{v}^{m, \text { tol }}$ into account. MSs allocated low-priority RBs however, exclusively sacrifice transmit power and, consequently, throughput. The allocation of users to these priority bands (i.e., the assignment of $x$-priority RBs to MSs) is discussed in Section 5.

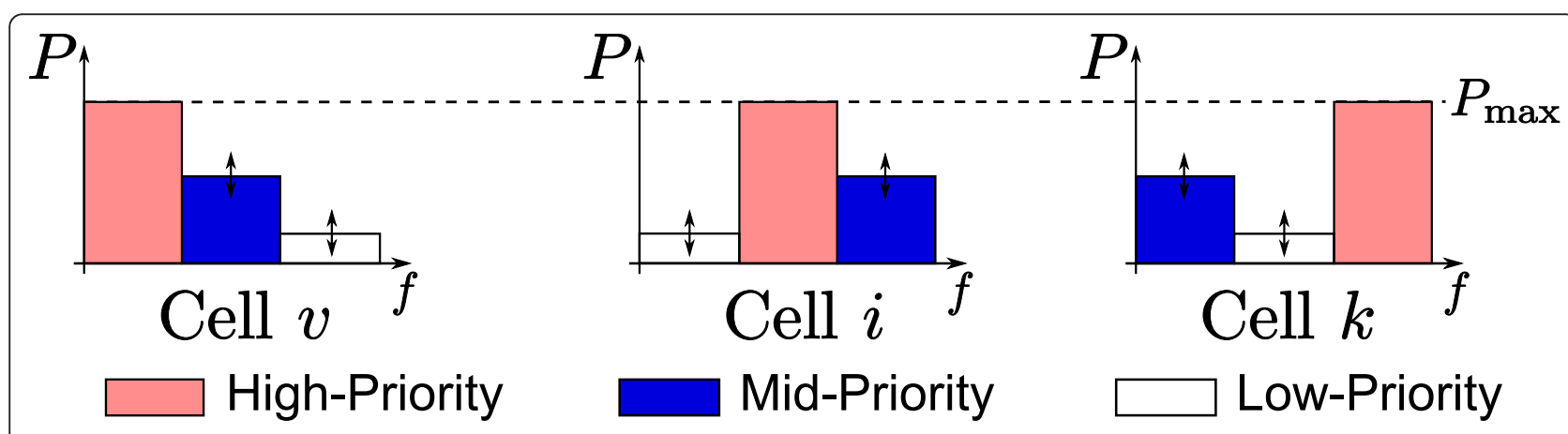

Figure 2 Allocation of priority bands in neighbouring cells $\boldsymbol{v}, \boldsymbol{i}$, and $\boldsymbol{j}$. The allocation of high-, mid- and low-priority RBs are complementary in the cells. 


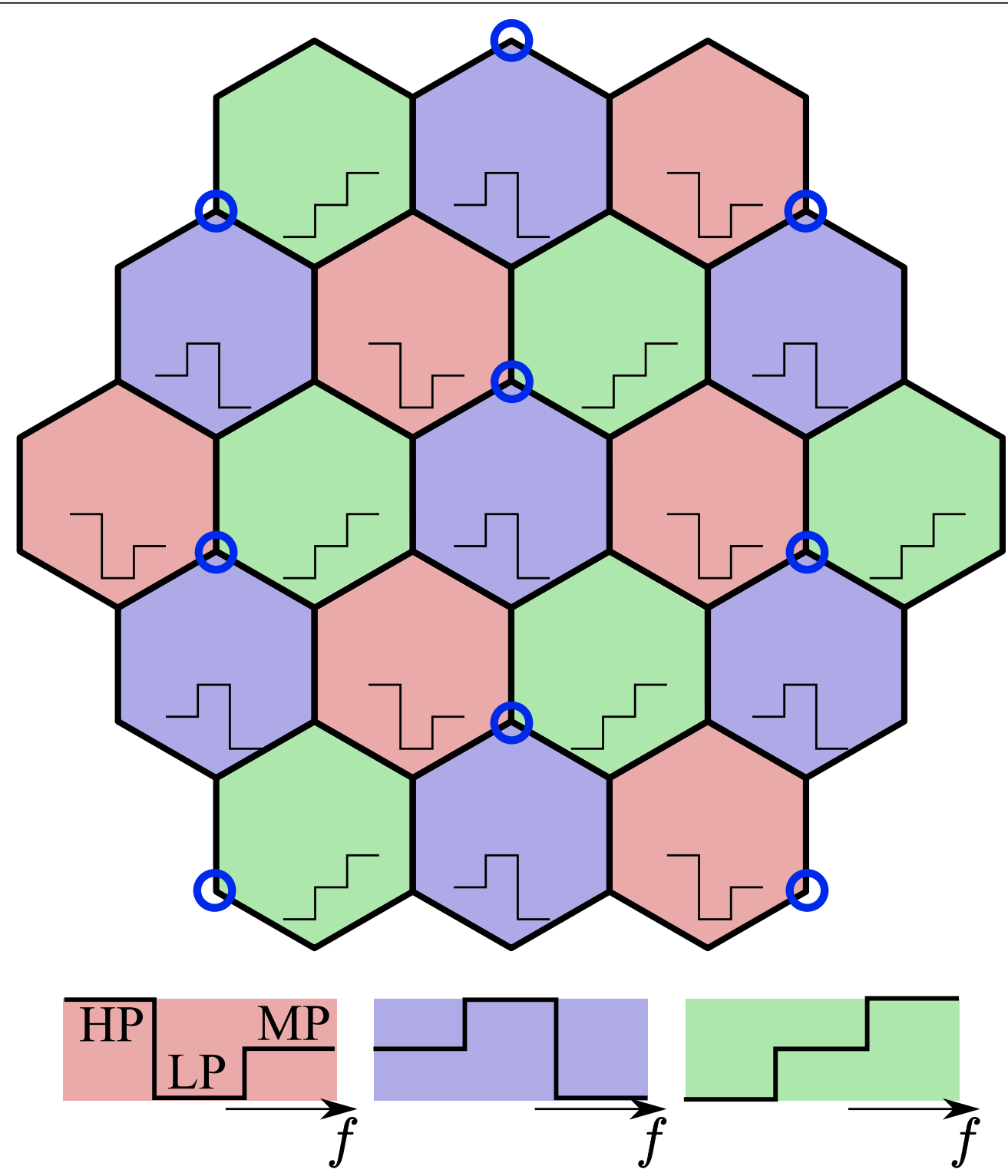

Figure 3 Allocation of priority bands in a multi-cellular network. The allocation of high-, mid- and low-priority RBs are complementary in the cells. A priority-class reuse scheme is arises. The colour bar indicates which part of the spectrum is given high-priority in which cells.

3.4. Practical implementation in LTE systems: an example In order to implement the ULIP procedure, the interfering (i.e., low-priority) MS needs to be informed of the $I_{v}^{m, \text { tol }}$ of its high-priority counterpart (in the neighbouring cell), to be able to then adjust its transmit power according to (12). This involves integrating the proposed ULIP technique within the network architecture. In abstract, the following procedure can be used to incorporate ULIP in the LTE network architecture:

(1) The vulnerable $\mathrm{BS}_{v_{v}}$ calculates the $I_{v}^{m, \text { tol }}$ for all (allocated) high-priority RBs in the cell using the received uplink desired signal strength $S_{v}^{m}$.
(2) The $I_{v}^{m, \text { tol }}$ are sent to all neighbouring BSs over the $\mathrm{X} 2$ or (if no X2 connection is available) S1 interfaces (see Figure 4 for LTE architecture).

(3) The neighbouring BS identifies and stores the minimum $I_{v}^{m, \text { tol }}$ received on each particular $\mathrm{RB}_{m}$, including the cell-ID from which it came.

(4) The neighbouring BS prepares a Data Radio Bearer (DRB) containing the $\min _{v_{v}}\left\{I_{v}^{m, t o l}\right\}$ found and the cell-ID $v_{v}$ for each of the low-priority RBs.

(5) The DRBs are sent with the Radio Resource Control (RRC) protocol via the Physical Downlink Shared 


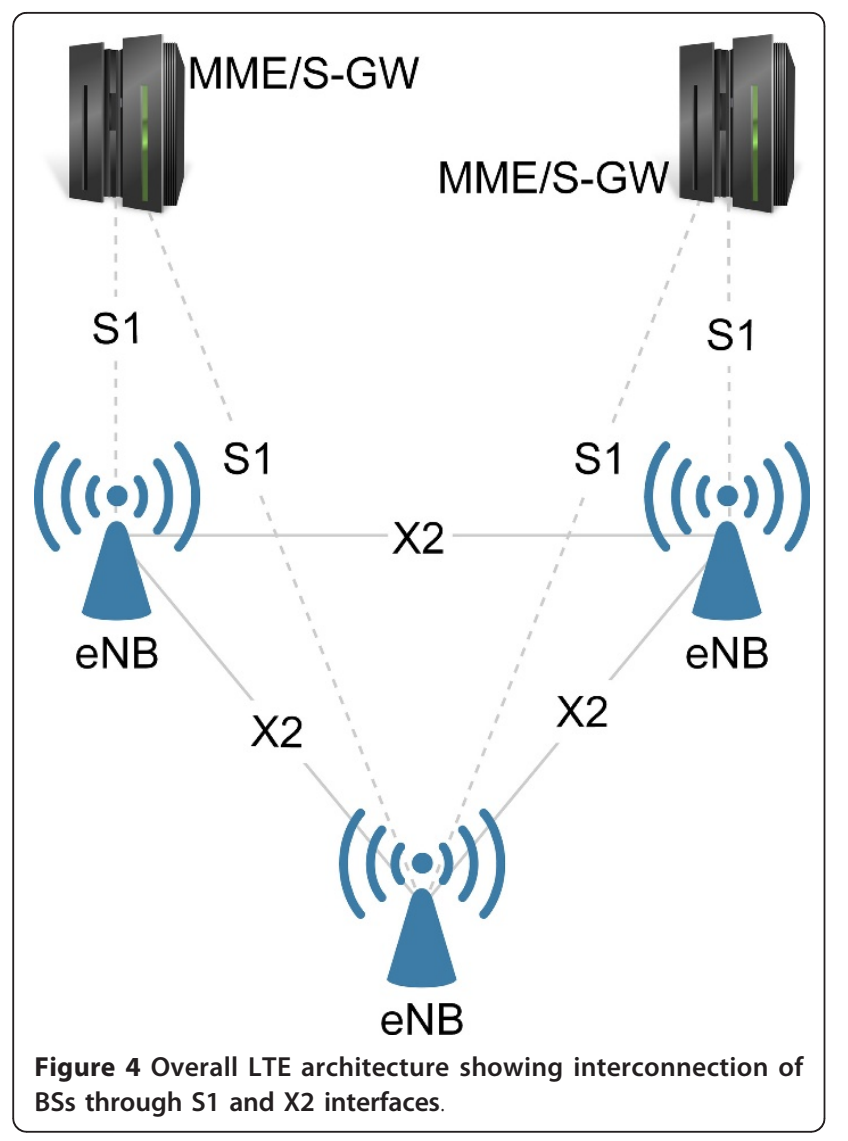

Channel (PDSCH) to each of the MSs allocated the lowpriority RBs (see Figure 5 for protocol).

(6) $\mathrm{MS}_{i}$ (allocated low-priority RB) estimates $G_{v_{v}, i}^{m}$ from BS $v_{v}$ with $\min _{v_{v}}\left\{I_{v}^{m, \text { tol }}\right\}$ indicated in DRB, using RSRP measurements.

(7) $\mathrm{MS}_{i}$ calculates $\tilde{P}_{\max , i}$ according to (12), and adjusts transmit power to provide interference protection in neighbouring cells.

The BS needs to inform the interfering $\mathrm{MS}_{i}$ of the interference margin $I_{v}^{m, \text { tol }}$ of $\mathrm{MS}_{v}$ on high-priority $\mathrm{RB}_{m}$ as calculated from (11). Thus, the transport of this information from BSs to the corresponding MSs must be defined using the LTE network architecture depicted in Figure 4.

The S1 interface connects the Serving Gateway (SGW)/Mobility Management Entity (MME) with groups of neighbouring BSs. The MME processes the signalling between an MS and the core network $(\mathrm{CN})$. Neighbouring BSs (i.e., within the groups connected by the $\mathrm{S} 1$ interface) are interconnected via the X2 interface, which carries control information regarding handover and interference coordination. The X2 interface is therefore highly suitable for ULIP related signalling.

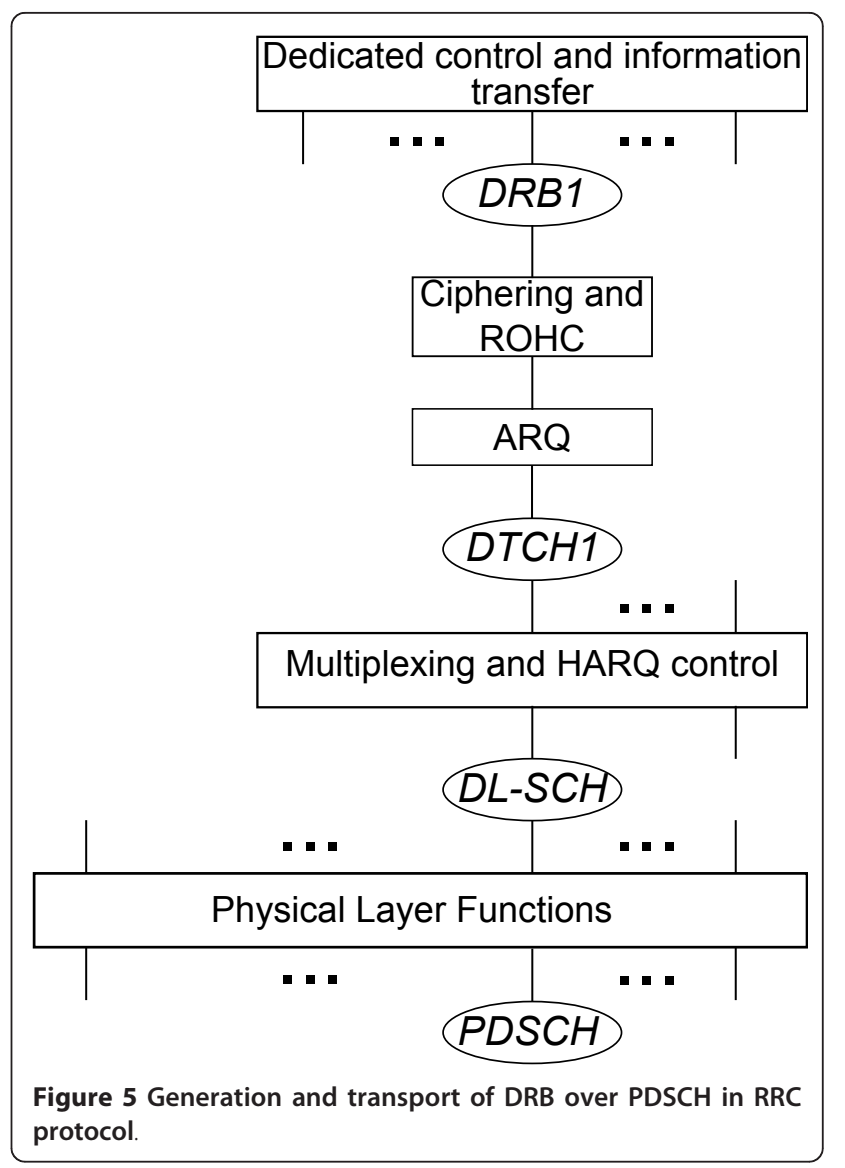

In LTE, the RRC protocol is used to transfer common (i.e., applicable to all MSs) and dedicated (i.e., applicable to only a specific MS) non-access stratum (NAS) information [27]. The RRC protocol covers a number of functional areas, including the broadcasting of system information, RRC connection control, network controlled mobility procedures, and measurement configuration and reporting. The RRC connection control handles all procedures related to the establishment, modification and termination of an RRC connection, including, among others, the formation of DRBs, radio bearers carrying user data [27].

In Figure 5, the construction, translation, and transmission of such a DRB is shown. Here, the DRB is multiplexed with other Signalling Radio Bearers (SRBs) and DRBs to then be transmitted to $\mathrm{MS}_{i}$. Furthermore, the Downlink Shared Channel (DL-SCH) and, consequently, the PDSCH are used for transmission of $\min _{v_{v}}\left\{I_{v}^{m}\right.$,tol $\}$ and the cell-ID, meaning that no extra signalling on the control channels is required. Of course, the transmission of these DRBs in every subframe would be highly signalling-intensive, and hence is to be avoided. While the serving BS will continuously update the $I_{v}^{m}$,tol for all 
high-priority RBs, it only transmits these updates to the neighbouring BSs when a significant change, $\delta$, in $I_{v}^{m, \text { tol }}$ in comparison to the last transmission (e.g., due to high mobility, call dropping, etc.) is achieved. This reduces the information transfer from the BSs to the MSs, and consequently lessens calculational intensity at the MSs.

Finally, knowledge of the cell-ID allows $\mathrm{MS}_{i}$ to read the cell-specific reference signals of the neighbouring BS providing $\min _{v_{v}}\left\{I_{v}^{m, \text { tol }}\right\}$, which is necessary to carry out the RSRP measurements and estimate the channel gain between the MS and the vulnerable BS, $G_{v_{v}, i}^{m}$. This, of course, is needed by the $\mathrm{MS}_{i}$ to perform its power adaptation according to (12). The RSRP for a specific cell is defined as the linear average over the power contributions of the resource elements, within the considered measurement frequency bandwidth, which carry the cell-specific reference signals [28]. Using these measurements, the power reduction procedure can take place.

\section{Performance analysis}

Given the detailed description of the ULIP technique, the expected performance of a system employing this mechanism can be explored. There are multiple analysis techniques that deal with such problems, more specifically with system capacity analysis. In [29-31], a reverse link capacity analysis assuming non-cooperative BSs (similar to the design of practical cellular systems) is unfortunately shown to be a long-standing open problem in information theory, but has been solved when treating the interference as Gaussian noise [32]. Clearly, since in ULIP the interference incident on each RB is dependent on the interference tolerances of other-cell high-priority MSs allocated that RB, the interference is most certainly not Gaussian. Hence, such an analysis is infeasible for a system employing ULIP. In $[29,33]$, the area spectral efficiency is introduced as a capacity measure that utilises stochastic geometry (statistical analysis of the positions and gains of MSs in the system) to estimate the expected capacity of a cellular network. Because in ULIP the users in a cell are split into three interdependent groups, such an analysis would be difficult as it is not always clear (by position) which MSs are assigned high-, mid-, or lowpriority. Furthermore, in [33] the interference is estimated stochastically, and since in ULIP the interference is dependent on individual MS requirements, this analysis would be misguided.

On the other hand, optimisation techniques [11,34] can be utilised to provide global solutions that optimise an overall performance goal (e.g., energy/spectral efficiency). Furthermore, these offer an overall characterisation of the wireless system. In ULIP, however, the aim is not to maximise/minimise any objective, but rather to provide individual MSs with the necessary interference mitigation such that these can achieve their SINR/rate requirements. This is clearly not a system-wide goal, and hence such a description of a ULIP system is not applicable.

In general, the main difficulty that is not overcome (in the aforementioned methods) is the multitude of interdependencies on each RB over the network. The transmit powers on an $R B$ are dependent on the signal qualities of the users allocated this RB in other cells in the network. Furthermore, these interdependencies are constantly adapting depending on the SINRs of the individual MSs in each cell. Hence, the stochastic interference modelling used in capacity analysis techniques cannot be utilised to model cellular ULIP. Therefore, a theoretical comparison to the state-of-the-art is performed to highlight the potential benefits of ULIP for OFDMA networks. And while transmit power control is standard for the reverse link in future systems, it has been shown that maximum power transmission is capacity-achieving [29], and thus this is compared to ULIP here. Analytical derivations for the energy efficiency and system capacity performance of ULIP are presented.

\subsection{Energy efficiency in ULIP}

In a system that employs ULIP, the transmit powers of low-priority MSs (MSs allocated low-priority RBs) are reduced so that interference to other cells is mitigated. Clearly, the throughput of the low-priority MSs is diminished relative to the reduction in transmit power. However, given a measure for energy efficiency, it can be shown that ULIP guarantees energy efficiency gains.

Given the metric for energy efficiency defined in (7):

$$
\beta_{u}=\frac{C_{u}}{P_{u}}=\frac{B_{u} \log _{2}\left(1+\gamma_{v}\right)}{P_{u}} \quad\left[\frac{\text { bits }}{\mathrm{J}}\right],
$$

it will be shown that the energy efficiency of $\mathrm{MS}_{u}$ after ULIP is applied is always greater than in the benchmark, where all MSs transmit at maximum power. Here, the Shannon capacity is used for ease of derivability and without loss of generality; and the calculation is performed independent of RBs, also with no loss of generality. Essentially, it will be shown that

$$
\beta_{u}^{\mathrm{ULIP}} \geq \beta_{u}^{\mathrm{BM}} \quad \text { or } \quad \frac{\beta_{u}^{\mathrm{ULIP}}}{\beta_{u}^{\mathrm{BM}}} \geq 1 .
$$

\subsubsection{Derivation}

The proof proceeds as follows

$$
\frac{\beta_{u}^{\mathrm{ULIP}}}{\beta_{u}^{\mathrm{BM}}}=\frac{B_{u} \log _{2}\left(1+\frac{P_{u}^{\mathrm{ULIP}} G}{I+N}\right) P_{u}^{\mathrm{BM}}}{B_{u} \log _{2}\left(1+\frac{P_{u}^{\mathrm{BM}} G}{I+N}\right) P_{u}^{\mathrm{ULIP}}},
$$


where $P_{u}^{\mathrm{BM}}$ is the benchmark transmit power, and $P_{u}^{\text {ULIP }}$ the power when ULIP is applied

$$
P_{u}^{\mathrm{ULIP}}=\alpha P_{u}^{\mathrm{BM}}, \quad 0 \leq \alpha \leq 1 .
$$

This is substituted into (14) to obtain (15)

$$
\begin{aligned}
\frac{\beta_{u}^{\mathrm{ULIP}}}{\beta_{u}^{\mathrm{BM}}} & =\frac{\log _{2}\left(1+\frac{\alpha P_{u}^{\mathrm{BM} G}}{I+N}\right) P_{u}^{\mathrm{BM}}}{\log _{2}\left(1+\frac{P_{u}^{\mathrm{BM}} G}{I+N}\right) \alpha P_{u}^{\mathrm{BM}}}, \\
& =\frac{\log _{2}(1+\alpha c)}{\alpha \log _{2}(1+c)}, \quad \text { where } c=\frac{P_{u}^{\mathrm{BM} G}}{I+N} .
\end{aligned}
$$

After rearranging (15) in the following manner

$$
\begin{aligned}
\frac{\log _{2}(1+\alpha c)}{\alpha \log _{2}(1+c)} & \geq 1 \\
\log _{2}(1+\alpha c) & \geq \alpha \log _{2}(1+c) \\
(1+\alpha c) & \geq(1+c)^{\alpha} .
\end{aligned}
$$

The generalised Bernoulli's inequality can be applied to prove the inequality in (17), which states

$$
(1+x)^{r} \leq 1+r x, \quad r \in \mathbb{R}, \quad 0 \leq r \leq 1, \quad x \in \mathbb{R}, x>-1 .
$$

To apply this to (17), $r$ and $x$ are set to

$$
\begin{aligned}
& r=\alpha, \quad 0 \leq \alpha \leq 1 \quad \rightarrow \quad 0 \leq r \leq 1 \\
& x=c, \quad c \geq 0 \quad \rightarrow \quad x \geq 0>-1
\end{aligned}
$$

and replaced in (18), such that

$$
\begin{aligned}
& (1+x)^{r} \leq 1+r x, \\
& (1+c)^{\alpha} \leq 1+\alpha c,
\end{aligned}
$$

exactly the inequality from (17). Hence, by proving (17), it has been shown that (13) is indeed true

$$
\frac{\beta_{u}^{\mathrm{ULIP}}}{\beta_{u}^{\mathrm{BM}}} \geq 1, \quad \forall P_{u}^{\mathrm{BM}} \geq P_{u}^{\mathrm{ULIP}} \geq 0,
$$

and hence it can be concluded that the energy efficiency of a low-priority MS employing ULIP is always greater than or equal to the energy efficiency of the same MS in the benchmark system (i.e., transmitting at maximum power). Furthermore, since MSs on highpriority RBs receive a capacity boost while maintaining transmit power, their energy efficiencies are also enhanced. Therefore, the energy efficiency of any/every MS in the system is augmented during ULIP operation, and consequently also the system energy efficiency

$$
\beta_{\text {sys }}^{\mathrm{ULIP}} \geq \beta_{\text {sys }}^{\mathrm{BM}} .
$$

For completeness, a similar proof can be constructed to show that in conjunction with a larger energy efficiency, the energy consumption $\psi_{u}=P_{u} / C_{u}$ [35], measured in $\mathrm{J} / \mathrm{bit}$, for ULIP is lower (as expected) than for the benchmark. Essentially,

$$
\frac{\psi_{u}^{\mathrm{ULIP}}}{\psi_{u}^{\mathrm{BM}}} \leq 1, \quad \text { and } \quad \psi_{\mathrm{sys}}^{\mathrm{ULIP}} \leq \psi_{\text {sys }}^{\mathrm{BM}}
$$

\subsection{System capacity in ULIP}

It has been shown that through the application of ULIP the energy efficiency of not only the individual MSs but also of the system is always improved (at minimum no losses are incurred). However, due to the reduction in overall system power through ULIP, one would expect, in general, a similar decrease in system capacity. Here it will be shown that this is not always the case, and hence ULIP not only guarantees a energy efficiency boost, but can also provide a gain in system capacity.

$$
C_{\text {sys }}^{\text {ULIP }} \nless C_{\text {sys }}^{\text {BM }}
$$

In essence, it is shown that (22) is true, which, combined with the energy efficiency results demonstrates the potential of ULIP for future OFDMA-based wireless networks such as LTE and/or LTE-Advanced. The proof is found in Appendix.

In the previous section it was demonstrated that the energy efficiency of any MS in a network will be enhanced when ULIP is employed, while here it has been shown that this energy efficiency boost can also be accompanied by an increase in the system capacity

$$
C_{\text {sys }}^{\mathrm{ULIP}} \nless C_{\text {sys }}^{\mathrm{BM}}, \quad \beta_{\text {sys }}^{\mathrm{ULIP}} \geq \beta_{\text {sys }}^{\mathrm{BM}} .
$$

Although in certain scenarios a loss in system capacity is incurred by the system-wide power reduction (as (22) suggests), the guaranteed energy efficiency gain can compensate this deficit. Furthermore, the possibility of gains in both performance metrics, i.e., when $C_{\text {sys }}$ is improved, is a good indication of the benefits ULIP can bring to future wireless networks.

\section{Scheduling}

To facilitate the interference protection, a scheduling procedure is designed to assign MSs to specific priority bands, enhancing the effect of ULIP in the system. In general, a random allocation of priority RBs can lead to undesired scenarios. For instance, the allocation of a high-priority RB to cell-centre MSs is wasteful, as such a MS-BS link is generally strong, and hence interference protection is unnecessary. At the cell-edge, allocating a low-priority RB to a MS is just as destructive. In this case, the MS will most probably be unable to sustain its 
$\gamma^{\mathrm{tar}}$, and hence fall into outage. Therefore, an appropriate scheduling mechanism is necessary for ULIP to achieve its full potential.

In a fair allocation scheme, cell-edge MSs should be allocated high-priority RBs so as to be able to transmit at full power and achieve the maximum possible SINR. Cell-centre users, which are more likely to achieve their SINR target due to BS proximity, should be assigned low-priority RBs. In essence, the general rule is to allocate high-priority RBs to the MSs with the least favourable SINR conditions.

Therefore, an efficient scheduling procedure can increase the effectiveness of ULIP, and prevent throughput losses due to MS outages. In this section, a scheduling procedure relying on the reverse link signals of the active users is presented. By analysing the signals, an approximation of the relative positions of the MSs (and their interferers) can be obtained, which can then be used to schedule the users accordingly. This presents a low complexity scheduling solution, as the necessary information is readily available at the BS.

\subsection{SINR scheduling}

The scheduling procedure utilises the SINRs from transmissions in previous time slots. In (23), $\mathcal{R}_{j}$ denotes the $N_{j}$-tuple of average (i.e., time average over the previous $z$ time slots, where $z$ is a system wide parameter) SINRs of the users in a cell

$$
\mathcal{R}_{j}=\left(\bar{\gamma}_{j, 1}, \bar{\gamma}_{j, 2}, \ldots, \bar{\gamma}_{j, N_{j}}\right),
$$

where $\bar{\gamma}_{j, i}$ is the average SINR (over all assigned RBs) of $\mathrm{MS}_{i}$ in cell $j$, and $N_{j}$ denotes the number of MSs in cell $j$. The MSs that are at the cell-edge experience, on average, weaker signals, and consequently low SINRs are received at their serving BS. Thus, the next step is to sort the $\bar{\gamma}_{j, i}$ in ascending order, so that the MSs that have the weakest SINRs can be identified

$$
\begin{array}{r}
\mathcal{U}_{j}^{*}=f_{\bar{\gamma}}\left(\mathcal{R}_{j}\right)=\left(p_{1}, p_{2}, \ldots, p_{N_{j}}\right) \\
\text { s.t. if } p_{k} \leq p_{l}, \text { then } \bar{\gamma}_{j, k} \leq \bar{\gamma}_{j, l},
\end{array}
$$

where $\mathcal{U}_{j}^{*}$ is the $N_{j}$-tuple of the positions $p_{k}$ of $\bar{\gamma}_{j, k}$ in the tuple $\mathcal{R}_{j}^{*}=\operatorname{order}\left(\mathcal{R}_{j}\right)$, which is sorted in ascending order. The function $f_{\bar{\gamma}}(\cdot)$ that defines this ordering can now be applied to the set of users in the cell of interest $\mathcal{S}_{\text {users }, j}$, and the set of high-priority MSs, $\mathcal{S}_{\mathrm{hp}, j}$, can be found

$$
\begin{aligned}
& \mathcal{S}_{\mathrm{hp}, j}=\left\{s \in \mathcal{S}_{\mathrm{users}, j} \mid f\left(\gamma_{j, s}\right) \leq\left\lceil\frac{N_{j}}{l}\right\rceil\right\} \\
& \text { where } \mathcal{S}_{\mathrm{hp}, j} \subset \mathcal{S}_{\text {users }, j},
\end{aligned}
$$

where $l$ denotes the number of priority bands such that the number of high-priority MSs yields $\left\lceil N_{j} / l\right\rceil$. In (25), the high-priority RBs are allocated to the $\left\lceil N_{i} / l\right\rceil$ MSs with the weakest average SINRs, and hence to the cell-edge. The low-priority RBs are allocated to the cellcentre, thus to the $\left\lceil\mathrm{N}_{\mathrm{j} / l}\right\rceil$ MSs with the strongest SINRs, and the mid-priority RBs to the remaining (middle set) MSs:

$$
\begin{gathered}
\mathcal{S}_{\mathrm{mp}, j}=\left\{s \in \mathcal{S}_{\mathrm{users}, j} \mid\left\lceil\frac{N_{j}}{3}\right\rceil \leq f\left(\gamma_{j, s}\right) \leq\left\lceil\frac{2 N_{j}}{3}\right\rceil\right\} \\
\mathcal{S}_{\mathrm{lp}, j}=\left\{s \in \mathcal{S}_{\mathrm{users}, j} \mid f\left(\gamma_{j, s}\right) \geq\left\lceil\frac{2 N_{j}}{3}\right\rceil\right\} \\
\text { where } \mathcal{S}_{\mathrm{mp}, j}, \mathcal{S}_{\mathrm{lp}, j} \subset \mathcal{S}_{\mathrm{users}, j} .
\end{gathered}
$$

One instance of the fair allocation for exactly $N_{j}=M$ $=50$ users per cell is depicted in Figure 6 .

It is clear to see that the farther MSs (from the serving $\mathrm{BS}$ ) have been allocated high-priority RBs, and to the nearer MSs, which are shielded from neighbouring cell interference, the low-priority RBs are assigned. The mid-priority RBs have been assigned to the remaining MSs.

When a new MS enters the cell, the initial allocation is performed using the SNR (which can be approximated using the RSRP), as no SINR information is available a priori. Mean SINR statistics are employed to eliminate fast fading effects and prevent a MS from rapidly changing priority class, so that the system can reach a stable operating point.

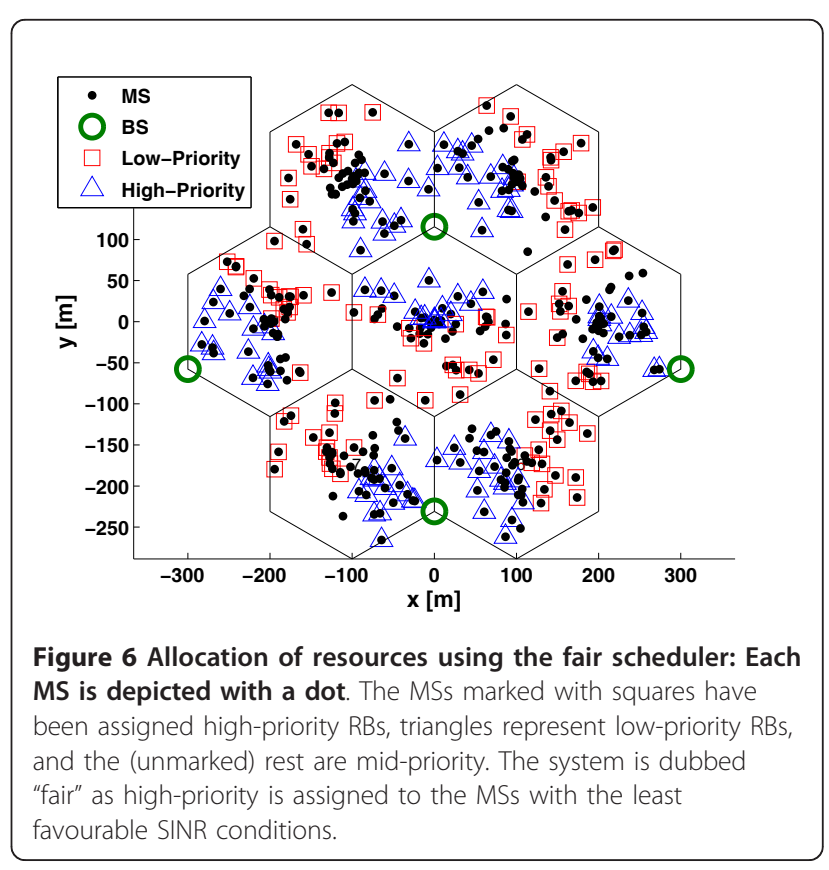




\section{Simulation}

Monte Carlo simulations are used to provide performance statistics of the users and the system with and without ULIP. The simulator is built following LTE specifications.

\subsection{Network construction and user distribution}

The simulation area is comprised of a single-tier, tessellated hexagonal cell distribution. To eliminate border effects with regards to interference, an additional two tiers are simulated. However, statistics are only taken from the first tier (and centre cell). Users are distributed uniformly over the simulation area such that each cell hosts, on average, $\bar{N}_{j}$ MSs. Further, BS-MS allocation is done based on path loss, such that each MS is assigned to the BS with the most favourable channel conditions.

Each cell is served by a sector of a macro-BS, where a $\mathrm{BS}$ has three $120^{\circ}$ sectors. Each BS is placed at the junction of the three hexagonal cells it serves. Figure 3 shows an example of the network construction and priority band allocation, and Figure 6 shows an example of the inner tier simulation area.

The (horizontal) azimuth antenna pattern, $A(\theta)$, is described by

$$
A(\theta)=-\min \left\{12\left(\frac{\theta}{\theta_{3 \mathrm{~dB}}}\right)^{2}, A_{m}\right\}
$$

where $\theta$ is the angle the MS-BS link deviates from the central lobe, $\theta_{3 \mathrm{~dB}}$ is the angle at which the gain is half that of at the centre of the lobe, and $A_{m}$ is the maximum possible attenuation [24]. Through (28), the horizontal signal attenuation due to MS position is determined.

\subsection{Resource allocation}

The priority classes in each cell are organised in the manner portrayed in Figure 3, such that when a MS is allocated to a particular priority class, its RBs (if it is assigned more than one) can be allocated contiguously, a feature particular to an LTE uplink. The allotment of users to priority classes is performed by the SINR scheduler introduced in Section 5. Within each class, the set of RBs is randomly (but still contiguously) allocated to the MSs assigned to that class, with each user receiving at minimum one RB.

\subsection{Time evolution}

Each run of the Monte Carlo simulation is iterated over $z=10$ subframes, or, equivalently, one LTE frame, such that long-term SINR statistics can be gathered. Due to the random user distribution, plentiful runs with different network generations are considered in order to obtain statistically accurate results. In each run, i.e., at the start of each subframe, the scheduling and allocation of RBs is reperformed. The MSs are assumed to be quasi-static for the duration of a run.

The simulation is performed for a full-buffer model, which represents the worst-case scenario where all users in the network are active, and no RB is left idle. Furthermore, the users are assumed to be static for the duration of a subframe, such that effects due to Doppler spread can be neglected. Perfect synchronisation in time and frequency is assumed, such that intra-cell interference is avoided. The relevant simulation parameters can be found in Table 2 .

\subsection{Benchmark}

To evaluate the performance of ULIP, two well-known benchmark systems have been implemented for comparison purposes. These are:

- Maximum power transmission: In the first benchmark, no power allocation is performed, and all MSs transmit at the maximum power on each RB.

- LTE power control: In the second benchmark, the transmit power is set dependent on the nominal SINR target $\Gamma$, the desired link path loss $L_{\text {des }}$, the strongest interfering link loss $L_{\text {int }}$, and the average interference received on that RB $I_{\text {avg }}^{m}$. Here, LTE fractional power control (FPC) [26] is used, where

$$
P_{\mathrm{dBm}}^{m}=\min \left\{\Gamma_{\mathrm{dB}}+I_{\mathrm{avg}, \mathrm{dBm}}^{m}+\alpha L_{\mathrm{des}, \mathrm{dB}}+(1-\alpha) L_{\mathrm{int}, \mathrm{dB}}, P_{\mathrm{max}, \mathrm{dBm}}\right\},
$$

Table 2 Simulation parameters

\begin{tabular}{ll}
\hline Parameter & Value \\
\hline Simulation area & 37 cells \\
Results area & inner 7 cells \\
Inter-site distance, $d_{\text {IS }} \bar{N}_{j}$ & $350 \mathrm{~m}$ \\
Average MSs per cell, & 20 \\
Uplink FDD band & {$[2.50,2.51] \mathrm{GHz}$} \\
Number of available RBs, $M$ & 50 \\
RB bandwidth, $B_{\mathrm{RB}}$ & $180 \mathrm{kHz}$ \\
Subcarriers per RB, $k_{\mathrm{sC}}$ & 12 \\
Symbol rate per subcarrier, $\mathrm{Q}_{\mathrm{s}}$ & $15 \mathrm{ksps}$ \\
Subframe duration, $t_{\mathrm{sf}}$ & $1 \mathrm{~ms}$ \\
Subframes (time slots), $z$ & 10 \\
Thermal noise, $\eta$ & $-174 \mathrm{dBm} / \mathrm{Hz}$ \\
Total MS transmit power & $23 \mathrm{dBm}$ \\
Sector width & $120^{\circ}$ \\
Sector $\theta_{3}$ dB & $70^{\circ}$ \\
MS SINR target, $\gamma^{\text {tar }}$ & $12 \mathrm{~dB}$ \\
Standard deviation, $\sigma$ & $4 \mathrm{~dB}$ \\
Auto-correlation distance & $50 \mathrm{~m}$ \\
\hline
\end{tabular}


which, depending on $\alpha$, achieves a balance between conventional power control $(\alpha=1)$ and maximum power transmission $(\alpha=0)$.

For each of the benchmarks, the RB allocation from the ULIP system is adopted, resulting in a soft frequency reuse scheme [36]. By comparing the performance of ULIP to these two benchmarks, the effect ULIP has on the performance of the system can be quantified.

\subsection{Results}

The performance of the system is measured by three criteria: achievable throughput, energy efficiency and fairness (as defined in (5), (7), and (8), respectively). Multiple iterations are run for a system employing ULIP and the benchmark systems. The cumulative distribution functions (CDFs) of achievable throughput and energy efficiency of individual MSs and of the network are compared. From this, quantitative average gain/loss statistics are generated.

\section{Results and discussion}

From the simulation, the CDFs of the achieved system throughput and energy efficiency are generated for systems employing ULIP and compared against the two benchmark systems, keeping the RB allocation unchanged. General simulation parameters are taken from Table 2 and [37], and full power control (i.e., $\alpha=$ 1 ) is implemented.

In Figure 7, the CDFs of the achieved user throughput for the three systems is shown, and it is evident that ULIP achieves considerable gains for MSs with low throughput in the benchmarks. At the 50th percentile, ULIP users achieve, on average, $2.8 \times$ the user throughput of both benchmarks.

Also, although at the 90th percentile a $31 \%$ loss is incurred by the power reduction on low-priority (and therefore high-throughput) RBs, the crossing point of the CDFs signifies that $82 \%$ of the users achieve a better SINR (and consequently throughput) in ULIP. Furthermore, the $\approx 20 \%$ outage seen in both benchmarks is eliminated, and hence ULIP provides significant advantages for the users in a cellular network.

These benefits are further seen in Figure 8, where the user energy efficiencies of the three systems are displayed. Here it is clear that ULIP provides a vast energy

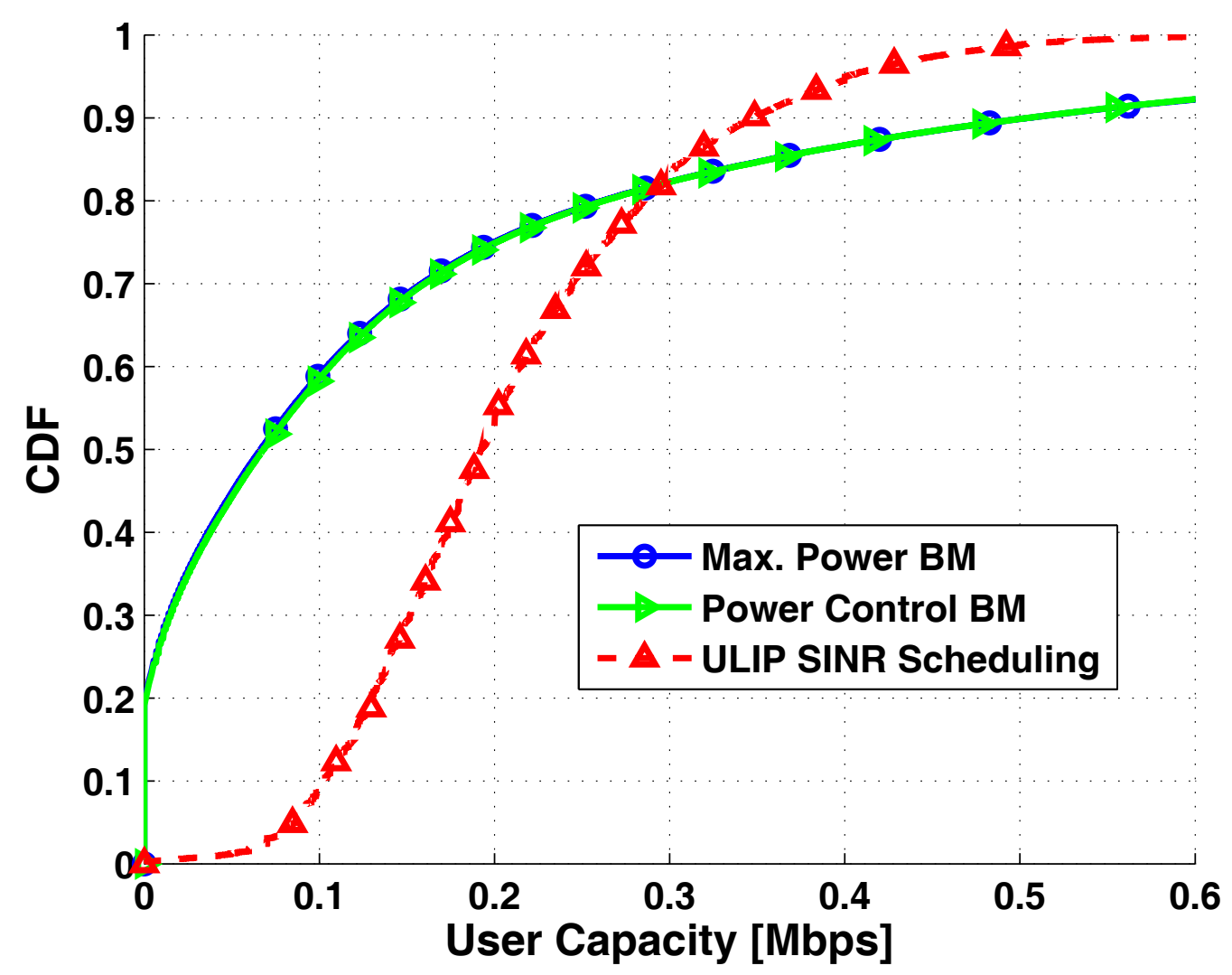

Figure 7 User throughput performance for ULIP and two benchmarks. System statistics are taken from the first tier (i.e., inner 7 cells) of the network over $z=10$ time slots, ULIP and power control $\gamma^{\text {ar }}=12 \mathrm{~dB}$. 


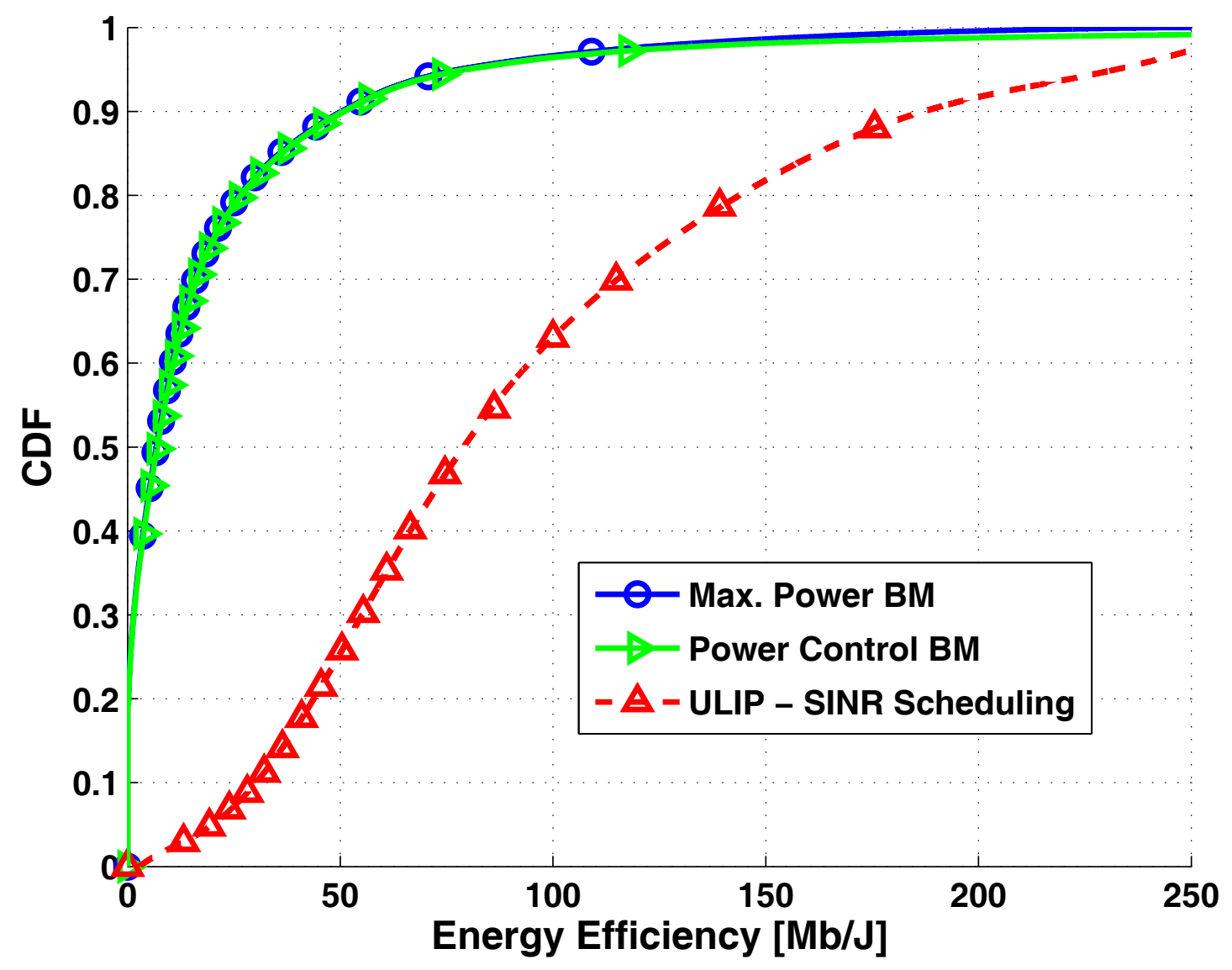

Figure 8 User energy efficiency performance.

efficiency improvement over the two benchmarks, which behave very similarly. At the 50th percentile, ULIP induces almost $11 \times$ the user energy efficiency of both benchmarks.

Furthermore, ULIP achieves energy efficiency gains for all MS over the maximum power benchmark, confirming the result of the performance analysis conducted in Section 4.1.

Figure 9 displays the system throughput fairness results of the three power allocation techniques. Here, it is clear to see that while power control provides some fairness gains (almost 14\%) over maximum power transmission, ULIP achieves by far the fairest system with over 0.8 fairness rating.

The substantial gains achieved by ULIP over maximum power transmission $(3.3 \times)$ can be accounted for by the balancing of the system capacity from the cellcentre to the cell-edge, boosting high-priority throughput by sacrificing that of the low-priority MSs, and hence achieving a more throughput fair system.
A further indicator of the enhanced fairness of the network is shown in Figure 10, where the MS throughput is plotted against the distance between the MS and its serving BS. And while both the maximum power and power control generate most of their capacity in the cell-centre (MSs closer to the BS), ULIP achieves an almost flat, much more even areal distribution of throughput in each cell. These findings confirm both the user throughput and fairness results shown in Figures 7 and 9, respectively. Furthermore, due to the simulation environment, the gains for many MSs are quite low, and hence power control very often utilises maximum transmit power to attempt to achieve the target SINR. Hence, there is little performance difference between the two systems, as is evident in Figure 10.

In Figure 11, the system throughput CDF results for ULIP, power control and maximum power transmission are shown. At the 50th percentile, it can be clearly seen that while power control surrenders a slight portion $(\approx$ $4 \%$ ) of the system capacity achieved by maximum power 


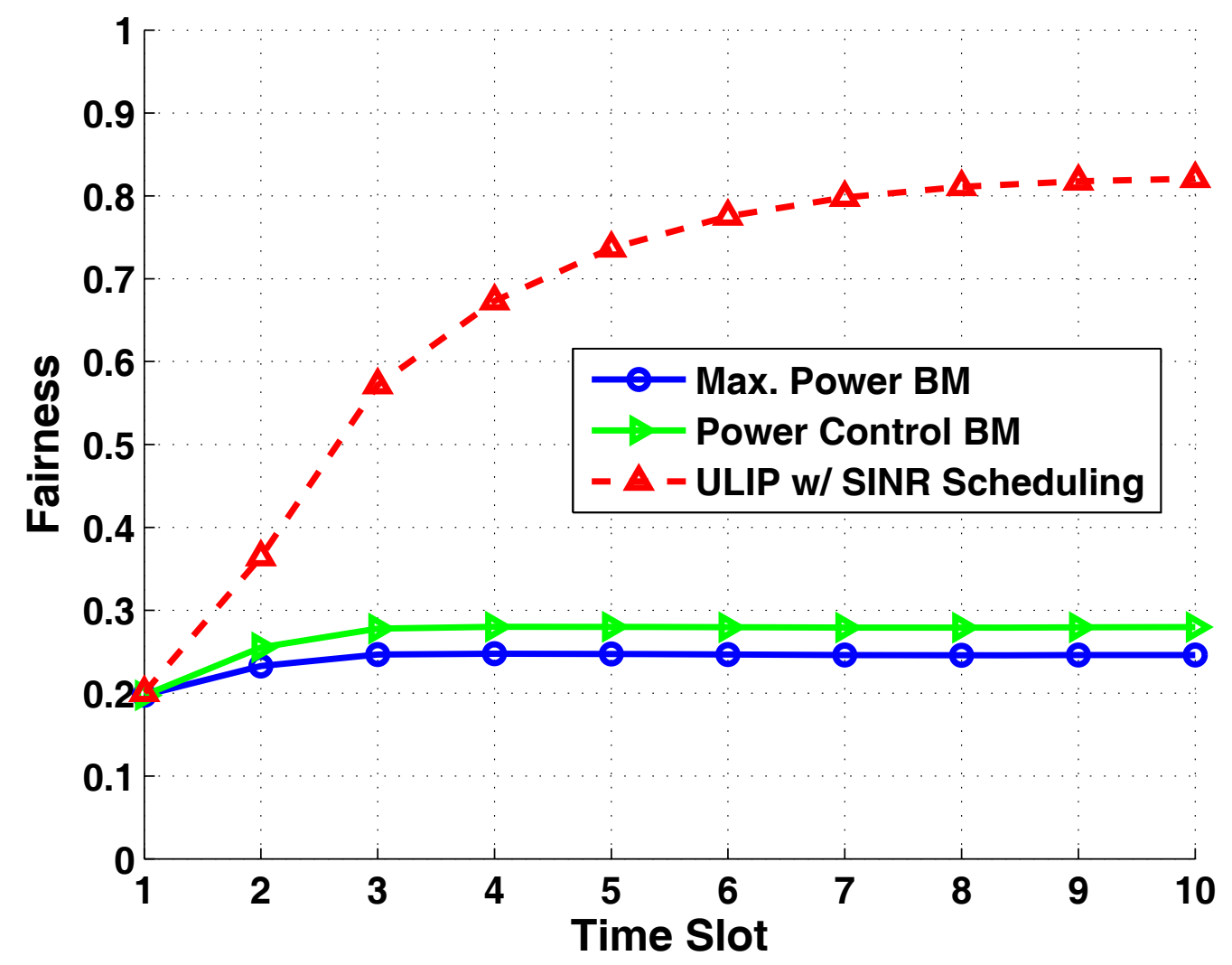

Figure 9 System throughput fairness performance.

transmission, ULIP produces a gain of over 15\%, resulting from the large number of MSs given throughput boosts (see Figure 7).

This is a very encouraging result, as it shows that the throughput shift from low- to high-priority MSs is beneficial for the system, achieving larger throughput gains for the high-priority users than losses by the low-priority MSs. This is also a direct result of the link adaptation, as any excess SINR (i.e., $\gamma>20 \mathrm{~dB}$ ) at the cellcentre can be transferred to the cell-edge without incurring any throughput losses for the low-priority (cell-centre) users. Furthermore, Figure 11 confirms the result achieved in Section 4.2, and shows further that system capacity gains are achievable.

In Figure 12, it can be seen that, surprisingly, power control exhibits an even worse energy efficiency than maximum power transmission. This is mainly due to the system throughput losses incurred. As expected, however, ULIP provides substantial gains over both benchmark systems, achieving a stout $3.5 \times$ and $3.6 \times$ the energy efficiency of max. power and power control at the 50th percentile, respectively. The large gains seen by ULIP are a combination of (a) the system throughput boosts achieved via the effective shifting of SINR from the cell-centre to the cell-edge; and (b) the substantial power reductions of the low- and mid-priority (cell-centre) users to protect the high-priority users from interference. Together, these two processes provide the significant energy efficiency gains seen in Figure 12, and confirm (20).

All in all, ULIP dominates each of the two benchmarks over the three performance criteria, especially providing a much more energy efficient and fair system. Furthermore, by achieving considerable gains in network capacity, it is clear that both performance analysis proofs have been confirmed.

\section{Summary and conclusions}

Full frequency reuse and the resulting large CCI in OFDMA networks brings forth the necessity for ICIC in future wireless networks. A technique for ULIP has been presented in this article, which provides protection from CCI through the power reduction of a subset of the neighbouring cell RBs, based on the SINR targets of the MSs in the cell of interest. Aside from the fact that no extra signalling is necessary over the control 


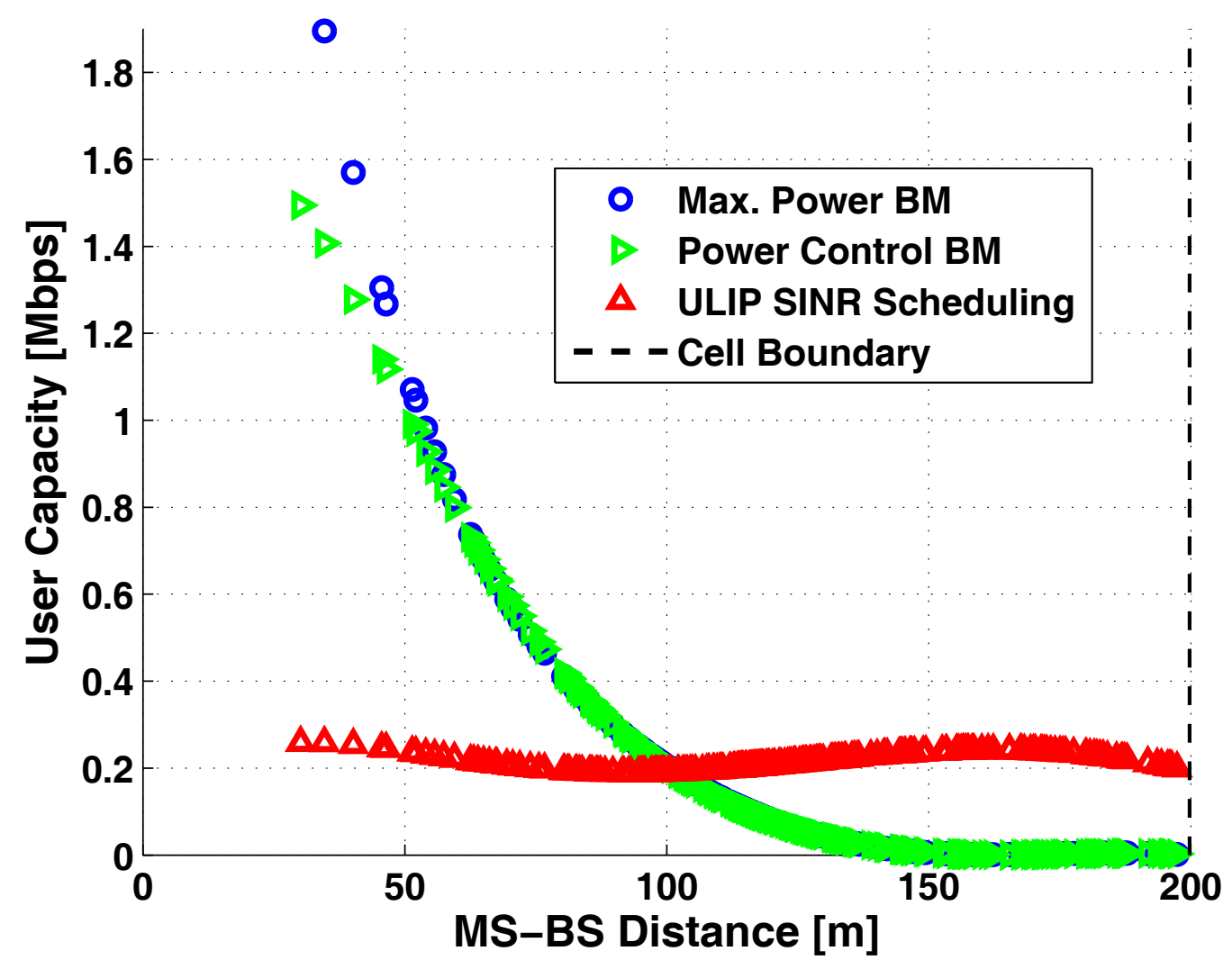

Figure 10 System throughput versus distance from serving BS.

channels, a further benefit of ULIP is a guaranteed increase in energy efficiency of all MSs in the system, and of the system as a whole. Furthermore, it was shown that while a loss in system capacity is possible, this is not certain, and hence gains in achievable system throughput are also possible. This is especially the case in networks where cell-edge capacity is limited, and most of the cell throughput is concentrated in the cellcentre.

It was shown that ULIP, combined with the SINR scheduler, achieves not only a $15 \%$ system capacity gain, but also substantially increases the system energy efficiency and fairness by $3.5 \times$ and $3.3 \times$, respectively. This is a direct result of the SINR displacement from the cell-centre to the cell-edge, and confirms the results in Section 4, highlighting the excellent energy efficiency of the ULIP protocol. A throughput drop is seen when power control is applied, mainly due to the SINR targeting of the system in comparison to maximum power, which does not restrict transmit power according to service requirements. Furthermore, ULIP eliminates the $\approx 20 \%$ outage suffered in the benchmarks, and provides throughput gains for over $80 \%$ of the MSs in the network. Consequently, ULIP diminishes the tradeoff between system capacity and fairness/energy efficiency, and provides significant gains in all three performance areas.

\section{Endnotes}

${ }^{a}$ In Table 1, the modulation and coding schemes are taken from LTE [27], and the SINR ranges from [38]. Here, the downlink values are used because no uplink implementation was found, as these values are operator specific. ${ }^{\mathrm{b}}$ These denote the priority status of the RBs within each class, and have no relation to user traffic priorities, which are not considered here.

\section{Appendix}

System capacity proof derivation

To prove (22), a counter-argument to the assumption that

$$
C_{\text {sys }}^{\mathrm{ULIP}} \leq C_{\text {sys }}^{\mathrm{BM}}
$$

must be found, where $C_{\text {sys }}$ is defined in (6). Therefore, a scenario is designed where the above assumption (30) 


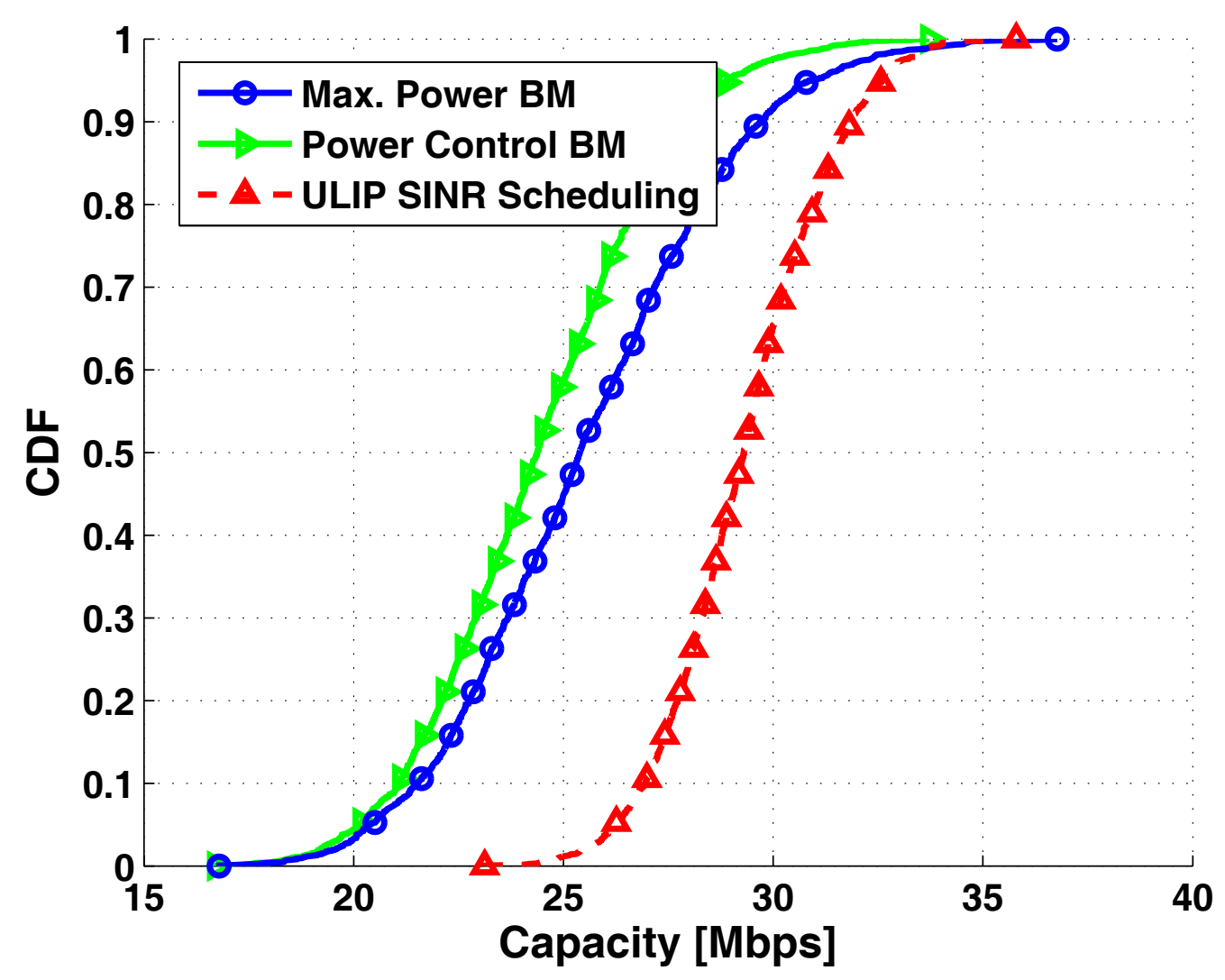

Figure 11 System sum throughput performance.

does not hold. A two-link scenario is chosen where $\mathrm{MS}_{1}$ and $\mathrm{MS}_{2}$ are allocated the same RBs in two neighbouring cells. Furthermore, we compare the $C_{\text {sys }}$ achieved in the benchmark (BM) system, in which all transmitting stations $\left(\mathrm{MS}_{1}\right.$ and $\left.\mathrm{MS}_{2}\right)$ transmit using maximum transmit power, to that achieved in the ULIP system. When ULIP is applied, $\mathrm{MS}_{2}$ is given high-priority, and $\mathrm{MS}_{1}$ low-priority status such that it may be required to scale it's power

$$
\begin{aligned}
\text { BM : } & P_{1}=P_{2} \\
\text { ULIP : } & \alpha P_{1} \leq P_{2}, \quad 0 \leq \alpha \leq 1
\end{aligned}
$$

where $\alpha$ is the scaling factor by which $\mathrm{MS}_{1}$ reduces its transmit power.

The proof is set up by making the assumption that the system is interference-limited, and hence the thermal noise can be ignored. This assumption depends on the inter-site distance $d_{\text {IS }}$ in the network, as clearly in larger cells the CCI diminishes (given $P_{\max }$ remains constant). The path gain and path loss equations are given by (9) and (10), respectively, and the thermal noise is calculated to be $\eta=k T B_{\mathrm{RB}}=-121 \mathrm{dBm}$, where $k$ is Boltzmann's constant, the temperature $T=300 \mathrm{~K}$, and the bandwidth $B_{\mathrm{RB}}=180 \mathrm{kHz}$ per RB. Given that, on average, $\left|H_{k, l}\right|^{2}=1$ and $X_{\sigma}=0$, the minimum average interfering link gain can be calculated when the interfering MS is located at the maximum distance $d_{\max }=d_{\mathrm{IS}}$ from the vulnerable BS (i.e., next to a neighbouring BS):

$$
\begin{aligned}
& L\left(d_{\max }\right)=15.3+37.6 \log _{10}(350)=110.9 \mathrm{~dB}, \\
& G_{\min , v_{2}}=10 \frac{-L(d)}{10}=-110.9 \mathrm{~dB} .
\end{aligned}
$$

And given $P_{u}=P_{\max } / M \approx 6 \mathrm{dBm}$, the minimum received interference is $P_{u} G_{\min , v_{2}} \approx-104.9 \mathrm{dBm}$, which is significantly larger than $\eta$. In fact, even for $d_{\mathrm{IS}}=500$ $\mathrm{m}$, the minimum average interference comes to -116.8 $\mathrm{dBm}$, which is still more than double the noise power.

Hence, assuming the network is constructed with $d_{\mathrm{IS}}$ $<500 \mathrm{~m}$, it has been shown that the system is interference-limited, and therefore the noise can be neglected. 


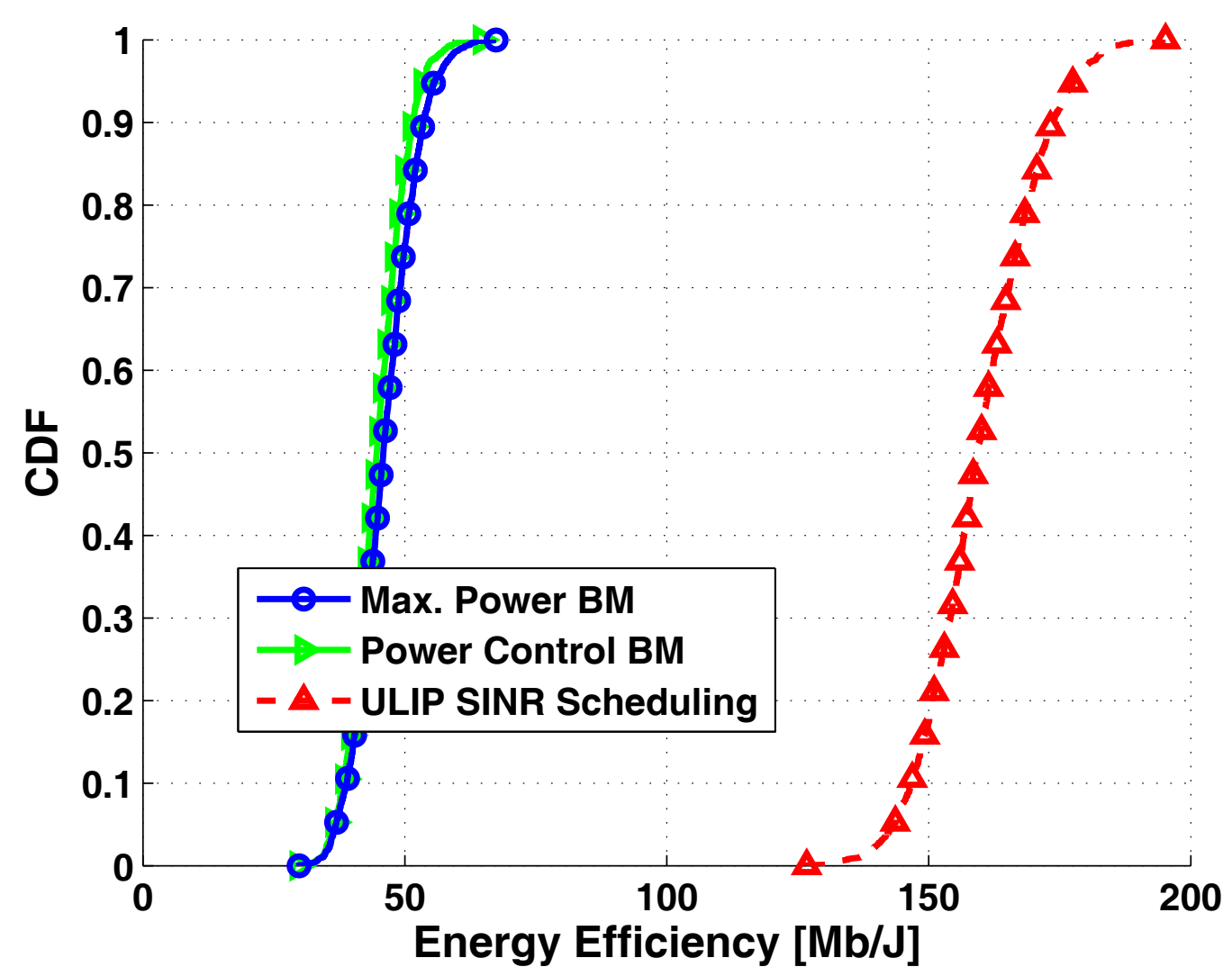

Figure 12 System energy efficiency performance.

This simplifies capacity calculations, as SIR can now be used rather than SINR. The individual user capacities are

$$
C_{1}^{\mathrm{BM}}=W \log _{2}\left(1+\frac{P_{1} G_{11}}{P_{2} G_{21}}\right) \quad C_{2}^{\mathrm{BM}}=W \log _{2}\left(1+\frac{P_{2} G_{22}}{P_{1} G_{12}}\right),
$$

in the benchmark system, and

$$
C_{1}^{\text {ULIP }}=W \log _{2}\left(1+\frac{\alpha P_{1} G_{11}}{P_{2} G_{21}}\right) \quad C_{2}^{\text {ULIP }}=W \log _{2}\left(1+\frac{P_{2} G_{22}}{\alpha P_{1} G_{12}}\right),
$$

when ULIP is employed, where Shannon's equation is used for the calculations. Subsequently, the relationship between $C_{i}^{\mathrm{BM}}$ and $C_{i}^{\mathrm{ULIP}}$ is found:

$$
C_{1}^{\text {ULIP }} \geq W \log _{2}\left(\left(1+\frac{P_{1} G_{11}}{P_{2} G_{21}}\right)^{\alpha}\right) \quad C_{2}^{\text {ULIP }} \geq W \log _{2}\left(\left(1+\frac{P_{2} G_{22}}{P_{1} G_{12}}\right)^{\frac{1}{\alpha}}\right)
$$

where Bernoulli's Inequality (18) is used to arrive at (35) (where $r=\alpha$ and $r=1 / \alpha$ for $i=1$ and 2, respectively). Further,

$$
C_{1}^{\text {ULIP }} \geq \alpha W \log _{2}\left(1+\frac{P_{1} G_{11}}{P_{2} G_{21}}\right) \quad C_{2}^{\text {ULIP }} \geq \frac{1}{\alpha} W \log _{2}\left(1+\frac{P_{2} G_{22}}{P_{1} G_{12}}\right),
$$

and finally, $C_{1}^{\mathrm{BM}}$ and $C_{2}^{\mathrm{BM}}$ are substituted into (36) to achieve (37)

$$
C_{1}^{\mathrm{ULIP}} \geq \alpha C_{1}^{\mathrm{BM}} \quad C_{2}^{\mathrm{ULIP}} \geq \frac{1}{\alpha} C_{2}^{\mathrm{BM}} .
$$

For further simplicity, let us assume that $G_{11}=G_{22}$, and $G_{12}=G_{21}$ (e.g., both MSs are at the cell-border). This creates the following set of equations:

$$
C_{1}^{\mathrm{BM}}=C_{2}^{\mathrm{BM}}=C_{i}^{\mathrm{BM}}, \quad C_{1}^{\mathrm{ULIP}} \geq \alpha C_{i}^{\mathrm{BM}}, \quad C_{2}^{\mathrm{ULIP}} \geq \frac{1}{\alpha}\left(\beta_{i}^{\mathrm{B} S}\right)
$$

Using (38), it can now be shown that the assumption in (30) does not hold for this system, and that hence (22) is true. In the benchmark,

$$
C_{\mathrm{sys}}^{\mathrm{BM}}=C_{1}^{\mathrm{BM}}+C_{2}^{\mathrm{BM}}=2 C_{i}^{\mathrm{BM}} .
$$

And when ULIP is applied,

$$
C_{\text {sys }}^{\mathrm{ULIP}}=C_{1}^{\mathrm{ULIP}}+C_{2}^{\mathrm{ULIP}}
$$




$$
\begin{aligned}
& \geq \alpha C_{1}^{\mathrm{BM}}+\frac{1}{\alpha} C_{2}^{\mathrm{BM}}=\left(\alpha+\frac{1}{\alpha}\right) C_{i}^{\mathrm{BM}}, \\
& \geq 2 C_{i}^{\mathrm{BM}}=C_{\text {sys }}^{\mathrm{BM}}
\end{aligned}
$$

where in (40), the equations from (38) are substituted into (39), and in (41), the inequality

$$
\left(\alpha+\frac{1}{\alpha}\right) \geq 2
$$

is used, which is proven by the inequality of arithmetic and geometric means

$$
\begin{aligned}
& \frac{a+b}{2} \geq \sqrt{a b}, \\
& a=\alpha, \quad b=\frac{1}{\alpha}, \quad\left(\alpha+\frac{1}{\alpha}\right) \geq 2 \sqrt{\alpha \frac{1}{\alpha}} \geq 2 .
\end{aligned}
$$

In (39)-(41) it has been demonstrated that for the chosen scenario, the ULIP system capacity is greater than that of the benchmark system

$$
C_{\text {sys }}^{\mathrm{ULIP}} \geq C_{\text {sys }}^{\mathrm{BM}}
$$

and that, hence, (30) is not true. Therefore, (22) is valid.

\section{Author details}

${ }^{1}$ Institute for Digital Communications, School of Engineering and Electronics, The University of Edinburgh, EH9 3JL, Edinburgh, UK ²DOCOMO Euro-Labs, Landsbergerstr. 312, 80687 Munich, Germany

\section{Competing interests}

The authors declare that they have no competing interests.

Received: 14 September 2011 Accepted: 28 May 2012

Published: 28 May 2012

\section{References}

1. G Auer, V Giannini, C Desset, I Godor, P Skillermark, M Olsson, M Imran, D Sabella, M Gonzalez, O Blume, A Fehske, How much energy is needed to run a wireless network? IEEE Wirel Commun. 18(5), 40-49 (2011)

2. D Astely, E Dahlman, A Furuskar, Y Jading, M Lindstrom, S Parkvall, LTE: the evolution of mobile broadband. IEEE Commun Mag. 47(4), 44-51 (2009)

3. M Alouini, A Goldsmith, Area spectral efficiency of cellular mobile radio systems. IEEE Trans Veh Technol. 48(4), 1047-1066 (1999). doi:10.1109/ 25.775355

4. S McLaughlin, PM Grant, JS Thompson, H Haas, DI Laurenson, C Khirallah, Y Hou, R Wang, Techniques for improving cellular radio base station energy efficiency. IEEE Wirel Commun Green Radio Special Issue. 18(5), 10-17 (2011)

5. G Boudreau, J Panicker, N Guo, R Chang, N Wang, S Vrzic, Interference coordination and cancellation for $4 \mathrm{G}$ networks. IEEE Commun Mag. 47(4), 74-81 (2009)

6. P Hosein, On uplink interference management for OFDMA networks, in Proc of the 18th International Symposium on Personal, Indoor and Mobile Radio Communications (PIMRC), Athens, Greece, 1-5 (Sept 2007)

7. M Al-Shalash, F Khafizov, Z Chao, Interference constrained soft frequency reuse for uplink icic in Ite networks, in Personal Indoor and Mobile Radio
Communications (PIMRC), 2010 IEEE 21st International Symposium, Istanbul, Turkey, 1882-1887 (Sept 2010)

8. W Xiao, R Ratasuk, A Ghosh, R Love, Y Sun, R Nory, Uplink power control, interference coordination and resource allocation for 3GPP E-UTRA, in Proc of the Vehicular Technology Conference (VTC), Montreal, Canada, 1-5 (Sept 2006)

9. T Zhang, Z Zeng, C Feng, J Cheng, L Song, Uplink power allocation for interference coordination in multi-cell OFDM systems, in Proc of the Third International Conference on Communications and Networking, Hangzhou, China, 716-720 (Aug 2008)

10. X Zhang, $\mathrm{C} \mathrm{He}$, L Jiang, J Xu, Inter-cell interference coordination based on softer frequency reuse in OFDMA cellular systems, in Proc of International Conference on Neural Networks and Signal Processing, Zhenjiang, China, 270-275 (June 2008)

11. L Miao, CG Cassandras, Optimal transmission scheduling for energy-efficient wireless networks, in Proc of 25th IEEE International Conference on Computer Communications, Barcelona, Spain, 1-11 (Apr 2006)

12. C Li, MJ Neely, Energy-optimal scheduling with dynamic channel acquisition in wireless downlinks. IEEE Trans Mobile Comput. 9(4), 527-539 (2010)

13. L Gao, S Cui, Efficient subcarrier, power, and rate allocation with fairness consideration for OFDMA uplink. IEEE Trans Wirel Commun. 7(5), 1507-1511 (2008)

14. M Al-Rawi, R Jantti, J Torsner, M Sagfors, Channel-aware inter-cell interference coordination for the uplink of 3G LTE networks, in Proc of the Wireless Telecommunications Symposium (WTS), (Prague, Czech Republic, Apr 2009), pp. 1-5

15. A Dejonghe, B Bougard, S Pollin, J Craninckx, A Bourdoux, LV der Perre, F Catthoor, Green reconfigurable radio systems. IEEE Signal Process Mag. 24(3), 90-101 (2007)

16. B Badic, T O'Farrrell, P Loskot, J He, Energy efficient radio access architectures for green radio: large versus small cell size deployment, in Proc of the 70th Vehicular Technology Conference, Anchorage, 1-5 (Sept 2009)

17. F Meshkati, V Poor, S Schwartz, Energy-efficient resource allocation in wireless networks: an overview of game theoretic approaches. IEEE Signal Proces Resource-Constrained Signal Processing, Communications and Networking Special Issue. 24(3), 58-68. (207)

18. C Xiong, G Li, S Zhang, Y Chen, S Xu, Energy- and spectral-efficiency tradeoff in downlink ofdma networks, in Communications (ICC), 2011 IEEE International Conference on, Kyoto, Japan, 1-5 (June 2011)

19. 3rd Generation Partnership Project (3GPP), Technical Specification Group Radio Access Network, Soft Frequency Reuse Scheme for UTRAN LTE, 3GPP TSG RAN WG1 R1-050507, 3GPP Std., 9-13 (May 2005)

20. R Jain, D Chiu, W Hawe, A Quantitative Measure of Fairness and Discrimination for Resource Allocation in Shared Computer Systems. DEC Technical Report, Tech Rep 301 (1984)

21. ITU-R Working Party 5D (WP5D) - IMT Systems, Report 124, Report of correspondence group for IMT.EVAL (United Arab Emirates, May 2008)

22. W Wang, T Ottosson, M Sternad, A Ahlen, A Svensson, Impact of multiuser diversity and channel variability on adaptive OFDM, in Proc of the 58th IEEE Vehicular Technology Conference (VTC), Orlando, USA, 547-551 (6-9 Oct 2003)

23. NTT DOCOMO, New Evaluation Models (Micro Cell, Indoor, Rural/HighSpeed), 3GPP TSG RAN WG1 R1-082713 (July 2008), http://www.3gpp.org/ ftp/tsg_ran/WG1_RL1/TSGR1_53b/Docs/. Accessed 27 Nov 2009

24. 3GPP, Simulation Assumptions and Parameters for FDD HeNB RF Requirements, 3GPP TSG RAN WG4 R4-092042 (July 2008), http:// www.3gpp.org/ftp/Specs/. Accessed 1 Sept 2009

25. LK Tee, C van Rensburg, J-A Tsai, Uplink power control for an OFDMA mobile cellular system, in Proc of the Vehicular Technology Conference, Baltimore, MD, 357-361 (Sept/Oct 2007)

26. A Rao, Reverse link power control for managing inter-cell interference in orthogonal multiple access systems, in Proc of Vehicular Technology Conference (VTC), Baltimore, MD, 1837-1841 (Sept/Oct 2007)

27. S Sesia, I Toufik, M Baker, in LTE-The UMTS Long Term Evolution: From Theory to Practice, ed. by S Sesia, I Toufik, M Baker 1st ed. (Wiley, Chichester, 2009)

28. 3GPP, Physical Channels and Modulation (Release 8), 3GPP TS 36.211 V 8.2.0 (2008-03) (Mar 2008), http://www.3gpp.org/ftp/Specs/. Accessed 1 Sept 2009 
29. A Goldsmith, Wireless Communications (Cambridge University Press, Cambridge, 2005)

30. TM Cover, JA Thomas, in Elements of Information Theory, ed. by DL Schilling 1st ed. (Wiley Series in Telecommunications Wiley, New York, 1991)

31. EC van der Meulen, Some reflections on the interference channel, in Communications and Cryptography: Two Sides of One Tapestry, ed. by RE Blahut, DJ Costello Jr., T Mittelholzer (Kluwer Academic Publishers, Boston, 1994), pp. 409-421

32. S Shamai, A Wyner, Information-theoretic considerations for symmetric, cellular, multiple-access fading channels. IEEE Trans Inf Theory. 43(6), 1877-1894 (1997). doi:10.1109/18.641553

33. Y Kim, T Kwon, D Hong, Area spectral efficiency of shared spectrum hierarchical cell structure networks. IEEE Trans Veh Technol. 59(8), 4145-4151 (2010)

34. Y Ma, D Kim, Rate-maximization scheduling schemes for uplink OFDMA. IEEE Trans Wirel Commun. 8(6), 3193-3205 (2009)

35. C Han, T Harrold, S Armour, I Krikidis, S Videv, P Grant, H Haas, J Thompson, I Ku, C-X Wang, TA Le, M Nakhai, J Zhang, L Hanzo, Green radio: radio techniques to enable energy-efficient wireless networks. IEEE Commun Mag. 49(6), 46-54 (2011)

36. X Mao, A Maaref, KH Teo, Adaptive soft frequency reuse for inter-cell interference coordination in SC-FDMA based 3GPP LTE uplinks, in Proc of the Global Telecommunications Conference, New Orleans, 1-6 (Nov 2008)

37. 3GPP, X2 General Aspects and Principles (Release 8), 3GPP TS 36.420 V8.0.0 (2007-12) (Dec 2007), http://www.3gpp.org/ftp/Specs/. Accessed 1 Sept 2009

38. EDX Wireless, Designing an LTE network using EDX SignalPro, Technical white paper (2010) retrieved 17 Jan 2011 http://www.edx.com/resources/ documents/

doi:10.1186/1687-1499-2012-180

Cite this article as: Burchardt et al: Uplink interference protection and scheduling for energy efficient OFDMA networks. EURASIP Journal on Wireless Communications and Networking 2012 2012:180.

\section{Submit your manuscript to a SpringerOpen ${ }^{\mathcal{O}}$ journal and benefit from:}

- Convenient online submission

- Rigorous peer review

- Immediate publication on acceptance

- Open access: articles freely available online

- High visibility within the field

- Retaining the copyright to your article

Submit your next manuscript at $\gg$ springeropen.com 